University of Massachusetts Amherst ScholarWorks@UMass Amherst

2004

\title{
Dense, Ionized, and Neutral Gas Surrounding Sagittarius $A^{*}$
}

H Shukla

Min Yun

University of Massachusetts - Amherst

NZ Scoville

Follow this and additional works at: https://scholarworks.umass.edu/astro_faculty_pubs Part of the Astrophysics and Astronomy Commons

\section{Recommended Citation}

Shukla, H; Yun, Min; and Scoville, NZ, "Dense, Ionized, and Neutral Gas Surrounding Sagittarius A*" (2004). The Astrophysical Journal. 1145.

$10.1086 / 424868$ 
submitted to the Astrophysical Journal

\title{
Dense Ionized and Neutral Gas Surrounding Sgr A*
}

\author{
Hemant Shukla ${ }^{1}$ \\ Owens Valley Radio Observatory, California Institute of Technology, Pasadena, CA 91125 \\ hemant@ovro.caltech.edu \\ Min S. Yun \\ University of Massachusetts, Astronomy Department, Amherst, MA 01003 \\ myun@astro.umass.edu \\ N. Z. Scoville \\ Owens Valley Radio Observatory, California Institute of Technology, Pasadena, CA 91125 \\ nzs@astro.caltech.edu
}

\begin{abstract}
We present high resolution $\mathrm{H} 41 \alpha$ hydrogen recombination line observations of the $1.2^{\prime}$ (3 pc) region surrounding Sagittarius $\mathrm{A}^{\star}\left(\mathrm{Sgr} \mathrm{A}^{\star}\right)$ at $92 \mathrm{GHz}$ using the Millimeter Array at the Owens Valley Radio Observatory (OVRO) with an angular resolution of $7^{\prime \prime} \times 3^{\prime \prime}$ and velocity resolution of $13 \mathrm{~km} \mathrm{~s}^{-1}$. New observations of H31 $\alpha, \mathrm{H} 35 \alpha$, $\mathrm{H} 41 \alpha$ and $\mathrm{H} 44 \alpha$ lines were obtained using the NRAO 12-m telescope, and their relative line strengths are interpreted in terms of various possible emission mechanisms. These NRAO 12-m measurements are the most extensive recombination line survey of this region to date. These OVRO data also represent the highest angular resolution and the highest sensitivity observations of Sgr A West in hydrogen recombination line and continuum emission at the millimeter $(\mathrm{mm})$ wavelengths. Observations of $\mathrm{HCO}^{+}(J=1 \rightarrow 0)$ transition at $89 \mathrm{GHz}$ are obtained simultaneously with a $40 \%$ improved angular resolution and 4-15 times improved sensitivity over the previously published results, and the distribution and kinematics of the dense molecular gas in the circumnuclear disk (CND) are mapped and compared with those of the ionized gas. The line brightness ratios of the hydrogen recombination lines are consistent with purely spontaneous emission from $T_{e} \sim 7000 \mathrm{~K}$ gas with $n_{e} \sim 2 \times 10^{4} \mathrm{~cm}^{-3}$ near LTE
\end{abstract}

\footnotetext{
${ }^{1}$ Present Address: Stanford Research Systems, 1290-C Reamwood Dr., Sunnyvale, CA, 94089; hshukla@thinksrs.com
} 
condition. A virial analysis suggests that the most prominent molecular gas clumps in the CND have mean densities of order $10^{7} \mathrm{~cm}^{-3}$, sufficient to withstand the tidal shear in the Galactic Center region. Therefore, these clumps may survive over several dynamical times, and the CND may be a dynamically stable structure. We estimate a total gas mass of about $3 \times 10^{5} M_{\odot}$ for the CND. Our combined analysis of the new high resolution $\mathrm{H} 41 \alpha$ and $\mathrm{HCO}^{+}$images and our kinematic model demonstrates a widely spread physical and dynamical link between the molecular gas in the CND and the ionized gas, including along the eastern rim of the CND where a gap was previously suggested.

Subject headings: Galaxy: center — galaxies: nuclei — ISM: molecules — radio continuum: ISM — radio lines: ISM

\section{Introduction}

Studying radio recombination lines (RRLs) is one of the most direct ways of probing ionized gas such as HII regions. Ionized gas properties such as kinematics, electron temperatures, and the geometrical filling factor can be directly constrained by the recombination line flux density, line width, free-free continuum flux density, and size of the line-emitting regions. At centimeter $(\mathrm{cm})$ wavelengths, RRLs are primarily stimulated emission amplifying extended non-thermal synchrotron emission. Therefore, distinguishing emission arising from diffuse, low density gas from that from clumpy, high density gas is difficult. Also, pressure broadening of the line width can complicate the interpretation of the cm RRL observations. For instance, cm RRL emission from luminous starburst systems such as Arp 220 may be dominated by internally stimulated emission from diffuse ionized gas (Anantharamaiah et al. 1993; Zhao et al. 1996) while spontaneous emission process is important at higher frequencies (e.g. Anantharamaiah et al. 2000; Yun et al. 2003). Interpretation of recombination lines at millimeter $(\mathrm{mm})$ wavelengths is more straightforward since stimulated emission and pressure broadening become negligible in most cases. Recent studies of mm RRLs in Galactic H II regions (Gordon \& Walmsley 1990; Martin et al. 1994; Gordon 1994) and starburst galaxies (Seaquist et al. 1996; Puxley et al. 1997) found higher line fluxes than expected from the lower frequency measurements. A steep rise in line flux density with frequency, characteristic of optically thin spontaneous emission, is seen in nearby starburst galaxies M82 and NGC 253 (Seaquist et al. 1996; Puxley et al. 1997).

The central 1 parsec region surrounding the putative central source in our Galactic Center, Sgr $A^{\star}$, is a frequent target of multi-wavelength investigations primarily because it is the nearest and the best laboratory for studying the active galactic nucleus (AGN) phenomenon and its fueling process. Currently this region appears to be relatively gas free. However, immediately surrounding Sgr $A^{\star}$ is an array of ionized gas streamers ("Sgr A West"; see review by Yusef-Zadeh et al. 2000). These streamers have been previously studied in the $12.8 \mu \mathrm{m}$ [Ne II] (Lacy et al. 1980; Serabyn \& 
Lacy 1985), P $\alpha$ (Scoville et al. 2003), and in cm RRL (Schwarz et al. 1989; Roberts et al. 1991; Roberts \& Goss 1993; Roberts, Yusef-Zadeh, \& Goss 1996), and they consist of several distinct kinematic features. The total mass of the ionized streamers is estimated to be less than $100 \mathrm{M}_{\odot}$ in total. Recent IR and radio observations found evidence for neutral gas within this central "cavity" (Genzel et al. 1985; Jackson et al. 1993; Latvakoski et al. 1999; Herrnstein \& Ho 2002), suggesting a more substantial presence of gas in the central parsec region. Surrounding and closely interacting with the ionized streamers is the dense and clumpy molecular ring commonly referred to as the circumnuclear disk (CND). The CND is asymmetric about $\mathrm{Sgr} \mathrm{A}^{\star}$, indicating its possible origin related to a gravitational capture of a passing cloud or energetic disruption of a stable ring (Morris \& Serabyn 1996). This ring is believed to represent a circumnuclear accretion disk feeding the inner parsec although some have argued that the CND is not a complete ring (see Marshall et al. 1995; Wright et al. 2001). The ionized streamers in Sgr A West are explained as cloudlets from the clumpy ring that after losing momentum have fallen toward the center and thereby have stretched and have been ionized by photo-dissociation within the inner parsec (see Sanders 1998). Sensitive imaging studies in cm RRL (Roberts et al. 1991) suggest high temperature $\left(T_{e}=5000-20,000\right.$ $\mathrm{K}$ ), with a possible radial temperature gradient increasing toward $\mathrm{Sgr} \mathrm{A}^{\star}$. The large proper motions of the stars very close to Sgr $\mathrm{A}^{\star}$ suggest central dark mass of 2.5-4.1 $\times 10^{6} \mathrm{M}_{\odot}$ (Genzel \& Eckart 1999; Ghez et al. 2000, 2003).

Motivated by the detection of extragalactic mm RRLs and recent new observations of the Galactic Center region, we have imaged the H41 $\alpha$ line at high angular resolution toward Sgr A* in order to study the nature of the ionized streamers and their relation to the dense neutral gas in and around the CND. At high frequencies, most of the RRL and continuum emission comes from the densest ionized gas (see Brown et al. 1978; Mohan et al. 2001). One of the primary goals of this study is to examine the existence of ionized gas denser than that traced by cm-wavelength RRLs in this region. The nature of the radiation mechanism is investigated by analyzing the new H31 $\alpha$, $\mathrm{H} 35 \alpha, \mathrm{H} 41 \alpha$, and $\mathrm{H} 44 \alpha$ line observations obtained at the NRAO 12-m telescope at Kitt Peak, Arizona. We further examine whether any dense neutral gas is directly associated with the ionized streamers using our sensitive, high resolution $\mathrm{HCO}^{+}(1-0)$ images. Our $\mathrm{HCO}^{+}(1-0)$ data have superior sensitivity and angular resolution over the previously published observations of similar molecular species (Güsten et al. 1987; Marr et al. 1993; Wright et al. 2001). In addition, a detailed analysis of the spatial distribution and kinematics of the dense molecular gas and ionized gas in the CND is offered using a simple kinematical model.

\section{Observations}

\subsection{OVRO Millimeter Telescope Array}

The $\mathrm{H} 41 \alpha$ and $\mathrm{HCO}^{+}(1-0)$ observations were made using the low, equatorial and high resolution configurations of the millimeter telescope array at Owens Valley Radio Observatory. The 
observational parameters from the multi-configuration observations conducted during the spring and fall 1998 are given in Table 1. The OVRO array consists of six 10.4 meter telescopes equipped with cooled SIS receivers. Interferometric measurements are made using a total of 45 independent baselines in order to maximize the sampling of the uv-plane and to achieve the highest imaging dynamic range possible. In the high resolution configuration the longest baseline used was 220 meters. The spectrometer modules were arranged so that each line was covered with a total of 60 channels at $4 \mathrm{MHz}\left(13 \mathrm{~km} \mathrm{~s}^{-1}\right)$ resolution centered at the velocity of $V_{L S R}=0.0 \mathrm{~km} \mathrm{~s}^{-1}$. By setting the LO frequency at $90.5 \mathrm{GHz}, \mathrm{H} 41 \alpha$ line at $92 \mathrm{GHz}$ (upper sideband) and $\mathrm{HCO}^{+}(1-0$ ) line at $89 \mathrm{GHz}$ (lower sideband) could be observed simultaneously. The primary beam (field of view) is about $85^{\prime \prime}$ at these frequencies. A nearby calibrator NRAO 530 was observed every 15 minutes to track the atmosphere and instrumental gain. The amplitude scaling is derived from the observations of $3 \mathrm{C} 273$, whose flux density is monitored daily relative to Uranus and Neptune with an accuracy of about $20 \%$.

The data were reduced using the OVRO data reduction program MMA (Scoville et al. 1993). Sgr $\mathrm{A}^{\star}$ is a low declination source, $\delta \sim-29^{\circ}$, and the synthesized beam is highly elongated in the N$\mathrm{S}$ direction. The resulting beam size is $6.95^{\prime \prime} \times 3.47^{\prime \prime}(\mathrm{FWHM})$. The data were imaged and analyzed using DIFMAP (Pearson et al. 1994) and Astronomical Image Processing System (AIPS) provided by the National Radio Astronomy Observatory (NRAO). Of the total 60 channels in the spectra, 15 line-free channels (60 MHz total) were used to construct the continuum image. Unresolved strong continuum source Sgr $\mathrm{A}^{\star}\left(2.0 \pm 0.3 \mathrm{Jy}_{\mathrm{beam}}{ }^{-1}\right)$ and the ionized streamers were used for self-calibration in DIFMAP, and the continuum was subtracted from the spectral line channels. All spectral and continuum images were corrected for primary beam attenuation. Subsequent analysis of the spectral line data cubes were conducted using AIPS and Groningen Image Processing System (GIPSY).

\subsection{NRAO 12-m Telescope}

We have obtained the H31 $\alpha$ (210.502 GHz), H35 $\alpha$ (147.047 GHz), H41 $\alpha$ (92.034 GHz), and $\mathrm{H} 44 \alpha(74.645 \mathrm{GHz})$ hydrogen recombination line spectra of Sgr A West using the NRAO 12-m telescope at Kitt Peak during May 1998. The NRAO 12-m telescope is equipped with single sideband SIS receivers, and typical system temperature ranged between 250 and $400 \mathrm{~K}$ (SSB), despite the low elevation of the source. The beam size is inversely proportional to the frequency and is $69^{\prime \prime}$ at $92 \mathrm{GHz}$. Both the filter banks (500 MHz at $2 \mathrm{MHz}$ resolution) and hybrid digital spectrometer $(600 \mathrm{MHz}$ at $0.78 \mathrm{MHz}$ resolution) were used to record the data. A wide range of OFF positions were examined, mostly along the minima in $\mathrm{CO}$ and $\mathrm{HCN}$ surveys of the Galactic Center region. After an extensive investigation, it was determined that the hydrogen recombination line emission is highly localized around Sgr $\mathrm{A}^{\star}$, and the final spectra presented here were obtained with a simple azimuth offset of $\pm 3^{\prime}$.

From the observatory report on the telescope efficiency measurements, we adopt the corrected 
main beam efficiency $\eta_{m}^{*}$ of $0.48,0.85,0.90$, and 0.95 for the four observed frequencies, respectively (Mangum 2000). These efficiencies are used to convert the observed brightness temperature $T_{R}^{*}$ to the main beam temperature $T_{m b}$ and then to flux density. The telescope efficiency measurements and the subsequent corrections are inherently uncertain, and we adopt an absolute uncertainty of $20 \%$ for the measured flux density.

\section{RESULTS}

\subsection{Continuum at $92 \mathrm{GHz}$}

The $92 \mathrm{GHz}$ continuum image of the Sgr A complex at the resolution of $6.95^{\prime \prime} \times 3.47^{\prime \prime}(\mathrm{PA}=$ $-5^{\circ}$ ) is shown in Figure 1. The major components of the Sgr A complex detected are the spiral structure known as Sgr A West and the compact central source Sgr A*, marked by a cross. The bright central source, Sgr $A^{\star}$, has peak flux density of $1.95 \pm 0.30 \mathrm{Jy}_{\text {beam }}{ }^{-1}$. The total $92 \mathrm{GHz}$ continuum flux density detected inside the $85^{\prime \prime}$ diameter region is $\sim 12 \mathrm{Jy}$. Some of the extended continuum emission may be missed by our observations as the 19-pointing mosaic image by Wright et al. (2001) reported a total flux of $\sim 15$ Jy covering a slightly larger area while Wright \& Backer (1993) reported a total flux of 27 Jy within a $2^{\prime}$ radius of Sgr $\mathrm{A}^{\star}$ from a combination of BIMA and Nobeyama 45-m telescope data.

The major continuum features of Sgr A West are identified in Figure 1, and their observed and derived properties are tabulated in Table 2. The east-west oriented filamentary structure located immediately south of Sgr A* is the bar of Sgr A West. The total continuum flux at $92 \mathrm{GHz}$ from the bar is about $0.9 \mathrm{Jy}$ with a peak brightness of $0.7 \mathrm{Jy} \mathrm{beam}^{-1}$. The bar appears to connect to the western arc and the eastern arc on each ends. Running almost perpendicular to the bar is the northern arm with higher total integrated flux density of about $1.1 \mathrm{Jy}$. The continuum flux is higher toward the southern part of the northern arm. To the west of the bar is the western arc that curves south. It also extends north almost reaching the northern arm. The continuum flux from the western arc is relatively weak. The eastern end of the bar curves along the eastern arm but is not detected further north. The morphology of the ionized gas around the Sgr A complex shown in Figure 1 is remarkably similar to the $8.3 \mathrm{GHz}$ continuum image by Roberts \& Goss (1993).

The peak flux density measured outside Sgr A* range between $0.1-0.75 \mathrm{Jy}^{\star}$ beam $^{-1}$ (see Table 2), which corresponds to peak brightness temperature of $1.1-6.0 \mathrm{~K}$. Assuming this continuum is largely thermal free-free emission from $7000 \mathrm{~K}$ gas (see $\S 4.2$ ), one can infer an optical depth of $\tau_{c} \lesssim 10^{-3}$ if the emitting region fills the beam with a unit filling factor. Even if the filling factor is not near unity, this inference of optically thin emission should be secure unless the continuum emission is entirely concentrated into discrete clumps with a diameter of 0.005 pc (1000 AU) or smaller. Continuum optical depth is given by

$$
\tau_{c}=0.0824 E M T_{e}^{-1.35} \nu_{G H z}^{-2.1}
$$


Assuming $T_{e}=7000 \mathrm{~K}$, the emission measure $\left(E M \equiv \int n_{e}^{2} d l\right)$ required to produce continuum opacity of $10^{-3}$ at $92 \mathrm{GHz}$ is $2.5 \times 10^{7} \mathrm{~cm}^{-6} \mathrm{pc}$. If the thickness of the filaments along the line of sight is comparable to the apparent width $(l \sim 0.1 \mathrm{pc})$, then the inferred mean electron density is $n_{e} \sim 2 \times 10^{4} \mathrm{~cm}^{-3}\left(N_{e} \sim 5 \times 10^{21} \mathrm{~cm}^{-2}\right)$. If the ionized gas is clumped at much smaller scales, then the electron density would be correspondingly larger. In comparison, Lutz et al. (1996) derived $n_{e} \sim 3000 \mathrm{~cm}^{-3}$ from their analysis of fine-structure line ratios. The consideration of collisional de-excitation of the observed [Ne II] emission places an upper limit on the electron density of about $10^{5} \mathrm{~cm}^{-3}$ (Jackson et al. 1993). Therefore the geometry of the ionized gas must be fairly smooth over $0.01-0.1$ pc scales.

\section{2. $\mathrm{H} 41 \alpha$}

The velocity integrated line flux density ("moment zero") image, shown in Figure 2, was generated by summing the continuum subtracted $\mathrm{H} 41 \alpha$ image cube over all velocity channels with line emission (from $-293 \mathrm{~km} \mathrm{~s}^{-1}$ to $+221 \mathrm{~km} \mathrm{~s}^{-1}$; see Figure 3 ). The cross marks the position of Sgr $A^{\star}$. The main features seen in the line emission map are very similar to the $92 \mathrm{GHz}$ continuum map shown in Figure 1, except for the absence of the bright, compact source corresponding to Sgr $\mathrm{A}^{\star}$. The emission from the western arc is weaker than seen in continuum. The northern arm is the brightest continuum feature besides $\mathrm{Sgr} \mathrm{A}^{\star}$, and the brightest $\mathrm{H} 41 \alpha$ emission region also occurs in the northern arm just northeast of Sgr $\mathrm{A}^{\star}$. The central bar shows a wide range of emission intensity and structures.

A summary of the measured parameters for all major features are given in Table 3 . The sizes tabulated for the listed regions have been calculated assuming $1^{\prime}=2.5 \mathrm{pc}$ (Reid 1989). Unlike the $92 \mathrm{GHz}$ continuum that peaks at Sgr $\mathrm{A}^{\star}$, the brightest feature in H41 $\alpha$ occurs in the northern arm with a peak brightness temperature of $\Delta T_{\text {peak }}=9.4 \pm 2.2 \mathrm{~K}$. The western arc, however, appears relatively weak in $\mathrm{H} 41 \alpha$ line. The peak line brightness for all $\mathrm{H} 41 \alpha$ are $\leq 10 \mathrm{~K}$, and thus the $\mathrm{H} 41 \alpha$ emission is also optically thin, $\tau_{L} \lesssim 10^{-3}$ (see below).

The spatially averaged $\mathrm{H} 41 \alpha$ line profiles for the six prominent emission regions (identified with boxes) are shown in Figure 4. The GIPSY tasks PROFIL and PROFIT were used to calculate the line-to-continuum (L/C) ratios and to fit a Gaussian profile to the data using a least squares procedure. The crosses indicate the data points, the solid lines are the Gaussian profiles, and the dashed lines represent the residuals. Calculations of Gaussian profiles yield four parameters: peak line-to-continuum flux density ratio, velocity integrated line intensity, central velocity, and the line width. These parameters are summarized in Table 4 along with the derived LTE electron temperature derived from the $\mathrm{L} / \mathrm{C}$ ratios (see further discussions in $\S 4.2$ ). A typical $\mathrm{L} / \mathrm{C}$ ratio along the northern arm is around 0.4 . The $\mathrm{L} / \mathrm{C}$ ratio is the largest $(\sim 0.9)$ in the western arc while the $\mathrm{L} / \mathrm{C}$ ratio in the bar drops from 0.6 to 0.03 within the 2 to 4 beam radii of $\mathrm{Sgr} \mathrm{A}^{\star}$.

The kinematics of the ionized gas traced by H41 $\alpha$ shown in Figures 3 and 4 are generally 
consistent with that of rotation about Sgr A*, similar to that of the CND, in broad agreement with previous studies of SgrA West in RRLs (Roberts et al. 1991; Roberts \& Goss 1993) and in 12.8 $\mu \mathrm{m}$ [Ne II] (Serabyn \& Lacy 1985). The central bar spans a very wide range of velocity, between $+33 \mathrm{~km} \mathrm{~s}^{-1}$ and $-293 \mathrm{~km} \mathrm{~s}^{-1}$ (see Figure 3), much larger than those associated with the northern arm (between $+163 \mathrm{~km} \mathrm{~s}^{-1}$ and $+7 \mathrm{~km} \mathrm{~s}^{-1}$ ) and the western arc (between $-7 \mathrm{~km} \mathrm{~s}^{-1}$ and -111 $\left.\mathrm{km} \mathrm{s}^{-1}\right)$. This large velocity gradient suggests that this gas is located deeper in the gravitational potential, much closer to the central mass concentration, than the rest of the ionized gas. Citing the relatively cool dust temperature along the bar, Latvakoski et al. (1999) have argued that the nearness of the bar to Sgr $\mathrm{A}^{\star}$ is largely in projection only, but this large spread in velocity and the correspondingly large velocity gradient requires that this gas feature has to be located much closer to the central mass concentration than the northern arm and the western arc. Since the CND is already viewed with a large inclination $\left(i \sim 60^{\circ}\right)$, placing a gas streamer in a more edge-on orbit can increase the line-of-sight velocity only by about $25 \%$. Therefore a viewing geometry alone cannot account for the large observed velocity gradient. Neither the angular resolution nor the overall S/N of the new H41 $\alpha$ data are better than the previous VLA H92 $\alpha$ measurements, and the main new insight obtained is that the ionized gas traced in $\mathrm{H} 41 \alpha$ emission share the same kinematics as the gas responsible for the $\mathrm{H} 92 \alpha$ emission.

The H41 $\alpha$ line widths of the ionized streamers range between $43 \mathrm{~km} \mathrm{~s}^{-1}$ and $347 \mathrm{~km} \mathrm{~s}^{-1}$ (see Table 4). The broadest line is seen toward Sgr $\mathrm{A}^{\star}$ with $\Delta \mathrm{V}=347 \pm 48 \mathrm{~km} \mathrm{~s}^{-1}$ (see Figure 4, Box 4 ), but the $\mathrm{S} / \mathrm{N}$ of this measurement is quite small in each individual channels. The next broadest line occurs in the vicinity of bright $\mathrm{H} 41 \alpha$ region in the northern arm with $\Delta \mathrm{V}=213 \pm 18 \mathrm{~km} \mathrm{~s}^{-1}$ (Box 6 in Figure 4). The narrowest line is seen toward the southern end of the western arc (Box 3 in Figure 4) with $\Delta \mathrm{V}=43 \pm 5 \mathrm{~km} \mathrm{~s}^{-1}$. Using $2^{\prime \prime}$ resolution VLA H92 $\alpha$ images, Roberts \& Goss (1993) have shown that crowding of distinct kinematical components along the line of sight can make the emission lines appear much broader than the intrinsic line width of about $50 \mathrm{~km} \mathrm{~s}^{-1}$. Combined with the large velocity gradient expected near the central mass concentration, the large line width we derive from $\mathrm{H} 41 \alpha$ emission must be primarily the result of spatial averaging resulting from our large observing beam of $7.0^{\prime \prime} \times 3.5^{\prime \prime}$. A typical line width measured in each pixel is between

40-75 $\mathrm{km} \mathrm{s}^{-1}$. Given the low $\mathrm{S} / \mathrm{N}$ of the data, it is not physically meaningful to deconvolve the individual kinematic components by fitting multiple Gaussians to the observed spectra. There is a clear trend of increasing L/C ratio with a decreasing line width. Such a trend is expected if the line and continuum emission arises from the same ionized gas clouds and the emitting gas has a narrow range of electron temperature (see $\S 4.2$ ).

\section{3. $\mathbf{H C O}^{+}(J=1 \rightarrow 0)$}

Figure 5 shows the high resolution image of the molecular ring in $\mathrm{HCO}^{+}$emission (grey-scale) superimposed over the $\mathrm{H} 41 \alpha$ (contour) emission. The circumnuclear ring traced in $\mathrm{HCO}^{+}$appears more complete than seen in the previous $\mathrm{HCN}$ (Güsten et al. 1987) and $\mathrm{HCO}^{+}$(Marr et al. 
1992) images obtained using the BIMA array. More recently Wright et al. (2001) imaged HCN, $\mathrm{HCO}^{+}$, and $\mathrm{H} 42 \alpha$ in a wider field of view using a 19-pointing mosaic, offering a much better overall picture of the molecular gas distribution in the $10 \mathrm{pc}$ region surrounding the Sgr $\mathrm{A}^{\star}$. However, the latest BIMA data by Wright et al. are not much better than the older BIMA data in resolution and sensitivity. These different images agree with each other broadly, but significant differences also exist in the details. One important difference is that the angular resolution of our new OVRO data is $40 \%$ better than those of Güsten et al. and Marr et al., and our sensitivity is also 4 and 15 times improved, respectively. The BIMA array is more sensitive to faint extended emission, and its data reduction procedure is tuned to emphasize such features. In contrast, our moment analysis is more sensitive to identifying bright emission clumps, and this accounts for the more complete appearance of the CND in Figure 5, consisting of many discrete features.

Determining whether any dense neutral gas is associated with the ionized streamers surrounding Sgr $\mathrm{A}^{\star}$ is one of the major objectives for our study. While the new observations have revealed clear evidence for a close physical link between ionized and neutral molecular gas (as discussed later in $\S 4.4), \mathrm{no} \mathrm{HCO}^{+}$emission is found spatially and kinematically coincident with the ionized streamers traced in $\mathrm{H} 41 \alpha$ (See Figure 3). Actually $\mathrm{HCO}^{+}$absorption is seen in many channels, particularly at blueshifted velocities, but most of these features show little kinematic relation to the $\mathrm{H} 41 \alpha$ emission. The spatial distribution of $\mathrm{HCO}^{+}$absorption resembles the entire continuum structure, and therefore the absorbing gas must be located in the foreground, associated with the $100 \mathrm{pc}$ scale molecular disk imaged by Tsuboi et al. (1999) and others. Among the redshifted velocity channels where little foreground absorption is present (e.g., +117 to $+195 \mathrm{~km} / \mathrm{s}$ ), there is little evidence for $\mathrm{HCO}^{+}$absorption at the locations of the $\mathrm{H} 41 \alpha$ and continuum emission peaks. In these channels, the upper limit to the optical depth for $\mathrm{HCO}^{+}$absorption is about $0.3(3 \sigma)$ at a spectral resolution of $13 \mathrm{~km} \mathrm{~s}^{-1}$. This limit corresponds to an upper limit on the $\mathrm{HCO}^{+}$column density of $4 \times 10^{12}$ $\mathrm{cm}^{-2}$. For an assumed $\mathrm{HCO}^{+}$abundance of $10^{-9}$, this translates into an $\mathrm{H}_{2}$ column density of $4 \times 10^{21} \mathrm{~cm}^{-2}$. If the $\mathrm{HCO}^{+}$abundance appropriate for a translucent cloud (where ion chemistry is important, see Hogerheijde et al. 1995) is adopted, the inferred upper limit on the $\mathrm{H}_{2}$ column density becomes as little as $\sim 10^{19} \mathrm{~cm}^{-2}$. These upper limits are close to or smaller than the column density of $N_{H} \sim(0.5-1.0) \times 10^{22} \mathrm{~cm}^{-2}$ inferred from the [O I]/[C II] far-IR line ratio (Jackson et al. 1993) and the mid-IR color analysis (Latvakoski et al. 1999). Using the calculations by Sternberg \& Dalgarno (1989), Jackson et al. (1993) have proposed that most of the molecules in the neutral gas inside the central cavity with $N_{H} \lesssim 10^{22} \mathrm{~cm}^{-2}$ are photo-dissociated and that the neutral gas is primarily atomic. Our $\mathrm{HCO}^{+}$absorption measurements are consistent with these conclusions, and we conclude independently that Sgr A West filaments are either highly ionized or are largely atomic. 


\section{4. $\mathrm{H} 31 \alpha, \mathrm{H} 35 \alpha, \mathrm{H} 41 \alpha$, and $\mathrm{H} 44 \alpha$}

The hydrogen recombination line spectra obtained at the NRAO 12-m telescope are shown in Figure 6, and the observing frequency and the measured line flux are summarized in Table 6. The mm frequency window is rich in spectral transitions, and studying faint emission lines in the Galactic Center region can be challenging. The H35 $\alpha$ line is not shown because it is completely swamped by the much stronger CS $J=3 \rightarrow 2$ line at $146.969 \mathrm{GHz}$, and no useful information could be derived.

The H44 spectrum, shown at the bottom of Figure 6, is the least confused and the highest $\mathrm{S} / \mathrm{N}$ measurement among all transitions observed at the $12-\mathrm{m}$ telescope. The observed line profile closely resembles the H41 $\alpha$ spectrum derived from the OVRO interferometer data (shown with a solid line in the middle of Figure 6). The total velocity integrated line flux density is $232 \pm 46 \mathrm{Jy}$ $\mathrm{km} \mathrm{s}^{-1}$ within the $84^{\prime \prime}$ beam of the $12-\mathrm{m}$ telescope at $74.645 \mathrm{GHz}$.

The H41 $\alpha$ spectrum obtained using the NRAO $12 \mathrm{~m}$ telescope, shown with a dotted line in the middle of Figure 6, shows a bright, narrow feature near $+200 \mathrm{~km} \mathrm{~s}^{-1}$. This is not seen in the H41 $\alpha$ spectrum obtained from the OVRO data (shown in solid line). We have mapped H41 $\alpha$ emission in a $2^{\prime}$ by $3^{\prime}$ region centered on $\mathrm{Sgr} \mathrm{A}^{\star}$ in order to examine the spatial extent of this spectral feature. The broad emission feature seen in the OVRO data and in the H44 $\alpha$ spectrum is spatially confined only to the central position while the bright, narrow feature appears everywhere with little velocity gradient. The narrow line width, little velocity gradient, and wide-spread distribution strongly indicate that this narrow component is a Galactic foreground emission. A likely identification is $\mathrm{CH}_{3} \mathrm{CN}$, which has transitions at 91.959, 91.970, 91.980, 91.985, and 91.987 GHz. The total velocity integrated $\mathrm{H} 41 \alpha$ line flux density from the OVRO data is $152 \pm 30 \mathrm{Jy} \mathrm{km} \mathrm{s}^{-1}$. This is a lower limit

since some extended flux may be missed by the interferometer (see below).

The H31 $\alpha$ spectrum shown at the top of Figure 6 is obtained by summing 8 independent spectra covering the whole Sgr A West complex. The telescope beam at $210.502 \mathrm{GHz}$ is about $30^{\prime \prime}$ and spatially resolves the Sgr A West structure. Therefore a $3 \times 3$ grid map was obtained at full beam sampling. The NE grid point was not observed, and the 8 spectra are summed together to derive the integrated H31 $\alpha$ spectrum. The H41 $\alpha$ channel maps (Figure 3) suggest that our $\mathrm{H} 31 \alpha$ spectrum may be missing some redshifted emission at $V=50-150 \mathrm{~km} \mathrm{~s}^{-1}$. Nevertheless, the summed spectrum closely resembles the $\mathrm{H} 41 \alpha$ and $\mathrm{H} 44 \alpha$ spectra, and we made no attempt to correct for any missing flux.

\section{DISCUSSION}

\subsection{Massive Compact Molecular Clumps in the CND}

The nine brightest $\mathrm{HCO}^{+}$clumps within the $\mathrm{CND}$ are identified as localized peaks of velocity integrated $\mathrm{HCO}^{+}$flux density map (Figure 5), and their physical sizes and emission characteristics 
are summarized in Table 5. The peak line brightness temperature, averaged over the $7^{\prime \prime} \times 3.5^{\prime \prime}(0.29$ $\mathrm{pc} \times 0.15 \mathrm{pc}$ ) beam, ranges between 10 and $30 \mathrm{~K}$. In comparison, the gas temperature derived from $\mathrm{NH}_{3}$ is $60-70 \mathrm{~K}$ (Coil \& Ho 2000) while the dust temperature of about $75 \mathrm{~K}$ is derived by Latvakoski et al. (1999) from the far-infrared observations. Since $\mathrm{HCO}^{+}$emission is strongly absorbed by the foreground gas along the line of sight and the beam filling factor is likely less than unity, the gas temperature inferred from the observed line brightness is strictly a lower limit. The measured peak $\mathrm{HCO}^{+}$line temperature is comparable to the values found by earlier BIMA studies (see $\S 3.3$ ) with a lower angular resolution, and one can safely deduce that the optically thick molecular gas nearly fills the beam at $\sim 0.1 \mathrm{pc}$ scales in these CND clumps.

We have computed virial masses of the individual $\mathrm{HCO}^{+}$emitting clouds as,

$$
M_{v i r}=250 r \Delta V^{2} M_{\odot},
$$

where $r$ is the radius of the cloud in pc and $\Delta V$ is the line width in $\mathrm{km} \mathrm{s}^{-1}$. If these individual molecular gas clumps are self-gravitating, then their masses range between $(0.3-4.5) \times 10^{4} M_{\odot}$ (see Table 5). Assuming a spherical geometry, the mean mass density $\left(\bar{\rho} \equiv M_{v i r} / \frac{4}{3} \pi r^{3}\right)$ is between $(1.3-10) \times 10^{-17} \mathrm{~g} \mathrm{~cm}^{-3}$ and mean $\mathrm{H}_{2}$ molecule density of $\bar{n}_{v i r}=(0.4-3.0) \times 10^{7} \mathrm{~cm}^{-3}$. These densities are significantly larger than the values inferred from the excitation analysis $\left(n \sim 10^{5-6}\right.$ $\mathrm{cm}^{-3}$, Genzel et al. 1985; Marr et al. 1993; Marshall et al. 1995). For a central source with a mass of $4.1 \pm 0.6 \times 10^{6} M_{\odot}$ (Ghez et al. 2003), the critical mass density required to survive the tidal shear at a radius of $1.5 \mathrm{pc}$ is $\bar{\rho}_{\text {tidal }}=4.3 \times 10^{-17} \mathrm{~g} \mathrm{~cm}^{-3}$ or $\bar{n}_{\text {tidal }}=2.5 \times 10^{7} \mathrm{~cm}^{-3}$. Contrary to the previous studies using excitation analysis, we conclude that the mean gas density inferred from the virial analysis is close to or exceeding the critical density for surviving the tidal shear if they are self-gravitating. Also, the CND may well be a structure that can survive several dynamical time scales ( $\gtrsim 10^{5}$ years). The total gas mass for the CND inferred from our virial analysis is around $3 \times 10^{5} M_{\odot}$, which is only a few per cent of the total dynamical mass for the central one parsec region. The virial mass of each of these clumps are about two orders of magnitude larger than that of the ionized gas associated with the whole ionized spiral (Sgr A West). Therefore, an infall of even a much smaller gas clump is sufficient to account for the observed ionized streamers. The new higher resolution 10-field mosaic observations of $\mathrm{HCO}^{+}$and $\mathrm{HCN}$ emission by Christopher et al. (2004) suggest even higher mean density $\left(\bar{n} \sim 10^{8} \mathrm{~cm}^{-3}\right)$ for these dense neutral clumps.

\subsection{Electron Temperature in Sgr A West}

The $92 \mathrm{GHz}$ continuum around Sgr A is primarily free-free emission, and a good spatial correspondence between the continuum (Fig. 1) and the H41 $\alpha$ line (Fig. 2) emission is naturally expected. Despite the similar morphology, however, a comparison suggests that the line emission is significantly clumpier than the continuum, especially in the western arc. The central bar is also clumpier in the line emission. In addition, in the northern arm the line emission appears brighter than anywhere else with an increasing trend toward Sgr A*. These differences may not be intrinsic 
to the source. A lower signal-to-noise ratio in the line image probably accounts for some of the differences. In addition, the continuum image suffers more from the missing flux problem than the line images because of a larger effective source size. ${ }^{1}$

For optically thin gas in LTE emitting thermal continuum and negligible pressure broadening, LTE electron temperature $T_{e}^{*}$ can be derived from the line-to-continuum ratio as,

$$
T_{e}^{*}=\left(6943 \nu^{1.1} \frac{S_{C}}{S_{L} \Delta V} \frac{1}{1+Y^{+}}\right)^{0.87}
$$

where $\nu$ is the observing frequency in GHz, $\Delta V$ is the velocity width (FWHM) in $\mathrm{km} \mathrm{s}^{-1}, S_{C}$ and $S_{L}$ are continuum and line flux densities, respectively, and $Y^{+}$is the singly ionized helium abundance (Brown et al. 1978; Roberts \& Goss 1993). In the case of Sgr A West the aforementioned assumptions may be reasonably made along with the interpretation that the continuum at $92 \mathrm{GHz}$ is largely free-free emission. The singly ionized helium abundance $Y^{+}$of $10 \%$ is assumed for Sgr A West (Roberts \& Goss 1993). The H41 $\alpha$ line widths listed in Table 4 are larger than the typical H92 $\alpha$ line widths of $40-50 \mathrm{~km} \mathrm{~s}^{-1}$ reported by Roberts \& Goss (1993), and this is the direct consequence of a poorer angular resolution of the H41 $\alpha$ observations. The resulting blending of emission features could conspire to produce unusual L/C ratios if gas temperature and density vary significantly along the ionized streamers. The high angular resolution observations by Roberts \& Goss (1993) have shown that this is not the case, and the poorer angular resolution of our H41 $\alpha$ observations should not affect our $T_{e}$ analysis in most cases.

An electron temperature map can be derived from the $92 \mathrm{GHz}$ continuum map (Fig. 1), the velocity integrated $\mathrm{H} 41 \alpha$ map (Fig. 2), and the relation given in Eq. 3. The derived electron temperature map, shown in Figure 7, reveals a fairly uniform distribution of $T_{e}$ around $7000 \mathrm{~K}$ throughout the Sgr A West complex except near Sgr A* (see Table 4). In general $T_{e}^{*}$ derived at higher frequencies tends to be larger because of diminishing contribution from stimulated emission. However, electron temperature derived with $\mathrm{H} 41 \alpha$ line at $92 \mathrm{GHz}$ agrees well with the derivation using H92 $\alpha$ line at $8.3 \mathrm{GHz}(7000 \pm 500 \mathrm{~K}$, Roberts \& Goss 1993), suggesting that the contribution from stimulated emission is negligible even frequencies as low as $8.3 \mathrm{GHz}$. Detecting little H41 $\alpha$ emission in the direction of the bright continuum source $\mathrm{Sgr} \mathrm{A}^{\star}$ is another clear evidence that stimulated emission is not important at $92 \mathrm{GHz}$ (see $\S 4.3$ ).

The higher electron temperature derived along the central bar just south of Sgr A* has previously been noted by Schwarz et al. (1989) and Roberts et al. (1991), and whether this is a real effect or an artifact of data analysis has been a subject of recent debates. Citing the discovery of highly blueshifted line emission near $-250 \mathrm{~km} \mathrm{~s}^{-1}$ missed by these earlier studies, Roberts \& Goss (1993) have suggested that the unusually low L/C ratio and thus the inferences of higher electron temperature may be erroneous. In general, a lower than expected L/C ratio can result from either

\footnotetext{
${ }^{1} \mathrm{~A}$ line emitting area is significantly smaller in size than the continuum emitting region in individual channel maps for an object with a large velocity gradient such as a rotating disk.
} 
(1) missing line flux or (2) extra continuum flux. The explanation by Roberts \& Goss (1993) is the former, but a careful analysis using the actual measurements of the extremely blueshifted line by Roberts, Yusef-Zadeh, \& Goss (1996) revealed that the missed flux alone cannot fully account for the low L/C ratio. In addition, Roberts, Yusef-Zadeh, \& Goss (1996) noted the presence of two optically thick continuum sources IRS 2 and IRS 13 whose additional continuum flux should contribute to the unusually low $\mathrm{L} / \mathrm{C}$ ratio (and higher $T_{e}$ ) observed. A broad coincidence of this position with that of the "mini-cavity" (Yusef-Zadeh et al. 1990) and its possible impact on the calculation of $T_{e}^{*}$ are discussed in detail by Roberts \& Goss (1993). Presence of significant structures at scales smaller than our synthesized beam violates the simple geometrical assumption going into Eq. 3 (also see Brown et al. 1978), and this may account for part of the observed local "enhancement" in $T_{e}^{*}$.

The local enhancement in electron temperature near the location of IRS 2 and IRS 13 is clearly seen in Figure 7 in the same manner as the seen in Figure 14 of Roberts \& Goss (1993). The persistence of this feature at $92 \mathrm{GHz}$ implies that the presence of the two continuum sources as suggested by Roberts, Yusef-Zadeh, \& Goss (1996) does not offer a full explanation for the observed low L/C ratio. Roberts, Yusef-Zadeh, \& Goss (1996) estimated an optical depth of $\sim 0.8$ and $\sim 1.1$ at $8.3 \mathrm{GHz}$ for the continuum sources IRS 2 and IRS 13 by comparing their $8.3 \mathrm{GHz}$ image with the 5 and $15 \mathrm{GHz}$ VLA continuum images. Free-free optical depth scales as $\tau \propto \nu^{-2.1}$ (see Eq. 1), and the continuum optical depth for IRS 2 and IRS 13 should be $\lesssim 0.01$ at $92 \mathrm{GHz}$. In comparison, optically thin free-free emission associated with the ionized filament should scale

only as $S_{\nu} \propto \nu^{-0.1}$. As a result, the continuum contribution from IRS 2 and IRS 13 should be substantially reduced at $92 \mathrm{GHz}$, and the $\mathrm{L} / \mathrm{C}$ ratio should reflect mostly the optically thin emission associated with the ionized gas filaments in SgrA West. However, the observed L/C ratio at 92 $\mathrm{GHz}$ is still noticeably lower at this location. The area of a lower $\mathrm{L} / \mathrm{C}$ ratio is not centered on these two continuum sources either. If continuum opacities for these two sources are much larger than estimated by Roberts et al. (i.e., optically thick even at $92 \mathrm{GHz}$ ), then the explanation of IRS 2 and IRS 13 as additional sources of continuum is still plausible, although the spatial distribution is still a problem. In summary, the apparent enhancement in $T_{e}$ (a low $\mathrm{L} / \mathrm{C}$ ratio) along the central bar just southwest of Sgr $A^{\star}$ is still not fully explained.

\subsection{Nature of the Recombination Line Emission}

The flux density ratio between $\mathrm{H} 41 \alpha$ and $\mathrm{H} 92 \alpha$ lines is shown in Figure 8. The overall structure of the northern arm, the bar, western arc, and eastern arm is almost identical at both frequencies, and the line ratio is nearly constant, $S_{H 92 \alpha} / S_{H 41 \alpha} \sim 0.15$. This similarity is remarkable given the order of magnitude difference in the frequencies of these lines. A slightly higher ratio is seen along the western arc and toward the top where the western arc arches into the northern arm, suggesting brighter emission at centimeter wavelengths. This trend of comparatively brighter emission at longer wavelength was also noted in the comparison of $\mathrm{H} 92 \alpha$ to $\operatorname{Br} \gamma$ ratios by Roberts \& Goss 
(1993). Roberts \& Goss offered extinction of $\operatorname{Br} \gamma$ emission by dust in the CND as a possible explanation, but a higher $S_{H 92 \alpha} / S_{H 41 \alpha}$ ratio would require a different explanation. Since the derived $T_{e}^{*}$ distribution is fairly constant (see Fig. 7 and Table 4 ), the enhanced $S_{H 92 \alpha} / S_{H 41 \alpha}$ ratio is not likely caused by any changes in the physical characteristics of the gas. The comparison of the total integrated $\mathrm{H} 41 \alpha$ line flux with those of other transitions measured with the NRAO $12-\mathrm{m}$ telescope suggest that some extended flux is missed by the interferometer (see $\S 3.4$ ). The shortest baseline in the OVRO observations of $3100 \lambda$ is about 4 times larger than that for the VLA observations $(830 \lambda)$, and the greater missing flux in the OVRO data offers a plausible explanation for the apparent enhanced $S_{H 92 \alpha} / S_{H 41 \alpha}$ ratio along the western arc.

The analysis of the $92 \mathrm{GHz}$ continuum (§3.1) and the H41 $\alpha$ emission (§3.2) suggests high electron density $\left(n_{e} \sim 2 \times 10^{4} \mathrm{~cm}^{-3}\right)$ and low optical depth $\left(\tau \lesssim 10^{-3}\right)$ for the ionized streamers. Under these conditions, both internal and external stimulated emission should be negligible, and optically thin spontaneous emission should dominate the recombination line emission at millimeter wavelengths. For the optically thin case near LTE, Eq. 3 implies that the velocity integrated line flux density $S_{L} \Delta V$ should increase linearly with frequency since $S_{C} \propto \nu^{-0.1}$ for thermal free-free emission. ${ }^{2}$ A plot of the velocity integrated line flux density for $\mathrm{H} 31 \alpha, \mathrm{H} 41 \alpha$, $\mathrm{H} 44 \alpha$, and $\mathrm{H} 92 \alpha$ lines is shown as a function of frequency shown in Figure 9, and indeed a nearly linear trend is seen. Excluding the H41 $\alpha$ measurement from the OVRO (which is a lower limit because of the missing flux), a best fit power-law slope is $\alpha=+0.95 \pm 0.18$, which is nearly exactly the theoretically expected value.

The assumption of "near LTE" is not strictly correct, however. A general expression for recombination line brightness in comparison to the LTE value is

$$
T_{L}=T_{L}^{*} b_{n}\left(1-\frac{1}{2} \tau_{c} \beta_{n}\right)
$$

where $T_{L}^{*}$ is the LTE line brightness, $b_{n}$ is the departure coefficient, $\tau_{c}$ is continuum optical depth, and $\beta_{n}$ is the effective line absorption coefficient accounting for non-LTE stimulated emission in the line. For the range of physical conditions we derive for the Sgr A West $\left(T_{e} \sim 7000 \mathrm{~K}, n_{e} \sim 10^{4}\right.$ $\mathrm{cm}^{-3}$ ), the computed values of these parameters are $b_{n} \sim 0.7-0.9$ and $\left(1-\beta_{n}\right) \sim 25$ (Brocklehurst 1970; Walmsley 1990). Although the non-LTE factor in the line absorption coefficient $\beta_{n}$ is large, it has little impact on the observed line brightness because a small optical depth $\left(\tau_{c} \lesssim 10^{-3}\right.$, as shown in $\S 3.1)$ makes the second term in Eq. 4 negligible, i.e. $\frac{1}{2} \tau_{c} \beta_{n} \sim 0$. The net effect is that the observed recombination line brightness is reduced by a factor of $b_{n}$ from the LTE value (i.e., $T_{L} / T_{L}^{*}=b_{n}$ ). The linear slope of the the observed power-law relation between the velocity integrated line flux density and frequency is unaffected as long as $\frac{1}{2} \tau_{c} \beta_{n} \sim 0$. As a result, the average flux density of the recombination line should increase linearly with frequency even when some non-LTE effects are present as long as spontaneous emission dominates the radiation process.

\footnotetext{
${ }^{2}$ Using slightly different assumptions, Seaquist et al. (1996) derive a relation showing $S_{L} \Delta V \propto \nu^{+2}$ in their Eq. A9. However, the $n^{3}$ factor, which they treated as a "constant" term going from their Eq. A8 to Eq. A9, actually behaves like $\nu^{-1}\left(\nu_{H n \alpha} \propto\left[1 / n^{2}-1 /(n+1)^{2}\right]\right)$, and this too leads to a linear relation, $S_{L} \Delta V \propto \nu^{+1}$.
} 
The comparison of the H41 $\alpha$ and H92 $\alpha$ spectra obtained toward Sgr A* (Figure 10) offers a definitive proof that the narrow $\mathrm{H} 92 \alpha$ line seen near $V=+50 \mathrm{~km} \mathrm{~s}^{-1}$ by Roberts \& Goss (1993) is indeed stimulated emission. Citing the nonthermal nature of the Sgr $\mathrm{A}^{\star}$ continuum emission and the low observed L/C ratio, Roberts \& Goss suggested that this H92 $\alpha$ emission must be stimulated emission arising from diffuse foreground ionized gas. The H92 $\alpha$ spectrum (shown in dotted line) and the H41 $\alpha$ spectrum (shown in solid line) have essentially identical shape at a position along the Northern Arm, $7^{\prime \prime}$ offset to the east of Sgr $A^{\star}$, suggesting the same ionized gas is responsible for both spectral features. At the location of $\mathrm{Sgr} \mathrm{A}^{\star}$, however, H92 $\alpha$ spectrum shows a distinct narrow component near $V=+50 \mathrm{~km} \mathrm{~s}^{-1}$, not seen in the H41 $\alpha$ spectrum. As shown in Eq. 1, the optical depth for H41 $\alpha$ line is expected to be more than 100 times smaller than the H92 $\alpha$ line, and $\mathrm{H} 41 \alpha$ is expected to be smaller by this large factor for optically thin stimulated emission.

\subsection{Physical Link between Neutral and Ionized Gas}

A review of the recent literature yields a coherent but complex picture for the composition, geometry, and kinematics of the neutral and ionized gas found in the central few parsec of our Galaxy. The $\mathrm{CND}$, traced in $\mathrm{HCO}^{+}$and $\mathrm{HCN}$, has a ring-like morphology with an inner radius of about $30^{\prime \prime}(1.25 \mathrm{pc})$ and an outer radius of at least $45-60^{\prime \prime}(1.8-2.5 \mathrm{pc})$ and is physically and kinematically linked with a larger scale disk with an extent of 10 pc or more (Wright et al. 2001; McGary et al. 2001; Christopher et al. 2004). It may be made of a multi-phase medium as the estimates of gas temperature $(50-250 \mathrm{~K})$ and density $\left(10^{4-6} \mathrm{~cm}^{-3}\right.$ and $\gtrsim 10^{7} \mathrm{~cm}^{-3}$ in dense clumps, see $\S 3.3$ ) range widely (Marr et al. 1993; Jackson et al. 1993; Marshall et al. 1995; Latvakoski et al. 1999). Within it lies the "central cavity" which actually contains the ionized streamers Sgr A West, made of about $100 M_{\odot}$ of $n_{e} \sim 10^{4} \mathrm{~cm}^{-3}$ gas at $T_{e} \sim 7000 \mathrm{~K}$ and as much as $1000 M_{\odot}$ of neutral gas which is thought to be mostly atomic with $n_{H} \sim 10^{5} \mathrm{~cm}^{-3}$ and $\mathrm{T}=100-300 \mathrm{~K}$ (Genzel et al. 1985; Roberts \& Goss 1993; Jackson et al. 1993; Telesco et al. 1996; Latvakoski et al. 1999, and see $\S 3.3)$. By obtaining the most sensitive and the highest angular resolution images of $\mathrm{H} 41 \alpha, 92 \mathrm{GHz}$ continuum, and $\mathrm{HCO}^{+}(1-0)$ to date simultaneously, we are able to shed some new light on the physical link between the neutral and ionized gas in this region.

The clearest demonstration of the physical connection between the neutral and ionized gas is the presence of recombination line peaks along the western arc, just inside of the dense gas clumps within the CND as shown by the overlay of the velocity integrated $\mathrm{H} 41 \alpha$ and $\mathrm{HCO}^{+}$images (see Figure 5). This radial offset between the ionized streamers and the molecular ring has been noted previously (Roberts \& Goss 1993), and it is consistent with the suggestion that the western arc is the ionized inner edge of the molecular ring directly exposed to a central ionizing source (Telesco et al. 1996). Evidence for a similar link between neutral and ionized gas is also seen on the eastern rim of the CND, between the eastern arm and the clump I shown in Figure 5. From their comparison of the $8 \mathrm{GHz}$ radio continuum map and the $30 \mu \mathrm{m}$ optical depth map, Telesco et al. (1996) postulated that the eastern arm is the ionized front of a protrusion of high-density 
material facing the central ionizing source. The clump I identified in Figure 5 may indeed be this previously unseen high-density material, revealed for the first time. The extinction map derived from the HST/NICMOS P $\alpha$ observations by Scoville et al. (2003) shows a hole in the obscuring material towards the northern arm and western arm, but high extinction regions with $A_{V} \geq 30$ surround this hole, including the HCN clump corresponding to our clump I. Possible ionizing source candidates range from the putative central engine Sgr A* to hot UV stars near the Galactic center (see discussion by Zhao et al. 1999). A more exotic possibility suggested by Maeda et al. (2002) is that the central source was much brighter in X-ray some 300 years ago as a result of the accretion of infalling material pushed in by the forward shocks from the supernova explosion in Sgr A East, leading to the ionization of the streamers.

A close physical link between the neutral gas in the CND and the ionized gas in Sgr A West is a widely occurring phenomenon, and this fact can be demonstrated more convincingly in the comparison of the channel maps as shown in Figure 3. Within the velocity ranges of $\pm 143 \mathrm{~km}$ $\mathrm{s}^{-1}$, the bulk of $\mathrm{H} 41 \alpha$ and $\mathrm{HCO}^{+}$features trace the same rotation-like kinematics about $\mathrm{Sgr} \mathrm{A}^{\star}$. More importantly, the $\mathrm{H} 41 \alpha$ features frequently occur just inside of the $\mathrm{HCO}^{+}$features (see the $V=-91,-39$, and $+65 \mathrm{~km} \mathrm{~s}^{-1}$ channels). As in the comparison of the velocity integrated images (Figure 5), this physical link is more evident along the western arc. There is a tendency for the $\mathrm{H} 41 \alpha$ features to show an azimuthal offset from the $\mathrm{HCO}^{+}$features, particularly for the features closer to Sgr $A^{\star}$ (e.g. $V=-39$ and $V=+65 \mathrm{~km} \mathrm{~s}^{-1}$ channels). These azimuthal offsets and a broad total line width $\left( \pm 200 \mathrm{~km} \mathrm{~s}^{-1}\right)$ for the $\mathrm{H} 41 \alpha$ line are naturally explained if the gas follows nearly Keplerian orbits around a highly concentrated mass distribution. (see the discussion in $\S 4.5$ ).

\subsection{Modeling Gas Kinematics in the CND}

Constrained by excellent high spatial resolution imaging data in $12.8 \mu \mathrm{m}$ [Ne II] and radio recombination lines, several plausible and detailed dynamical models for the ionized streamers have been constructed (e.g. Sanders 1998; Vollmer \& Duschl 2000). Models of gas kinematics for the CND have also been constructed, mostly to explain the features identified in low resolution images $\left(\theta \sim 10-20^{\prime \prime}\right)$ that are susceptible to confusion with ubiquitous foreground and background emission (e.g. Liszt et al. 1985; Marshall et al. 1995). Taking advantage of the new high (and matched) resolution spectroscopic imaging data in $\mathrm{H} 41 \alpha$ and $\mathrm{HCO}^{+}$, we can now examine in detail the gas kinematics and the gravitational potential in the central few parsec radius region in a self-consistent manner. We have already concluded that some of the ionized gas is orbiting Sgr $\mathrm{A}^{\star}$ in the plane defined by the CND (see $\S 4.4$ ). Using the velocity information, we can also isolate and examine other kinematic features proposed to be associated with the CND, such as the " +50 $\mathrm{km} \mathrm{s}^{-1}$ streamer" and the "+70 $\mathrm{km} \mathrm{s}^{-1}$ feature" (see Jackson et al. 1993).

If a massive central source $\left(2.5-4.1 \times 10^{6} \mathrm{M}_{\odot}\right.$, Genzel \& Eckart 1999; Ghez et al. 2000, 2003) dominates the gravitational potential in the central parsec region, the kinematics of the surrounding gas disk should be regular and well behaved. The neutral and ionized gas inside the 
CND contributes only about $1000 M_{\odot}$ while we estimate the total mass of the CND to be about $3 \times 10^{5} M_{\odot}($ see $\S 4.4)$. The mass of the central stellar cluster is more substantial and is highly centrally concentrated, doubling the enclosed total mass near 2 pc radius (Genzel et al. 2003). We adopt a mass distribution of the form similar to Eq. 3 of Vollmer \& Duschl (2002), after updating it using the most recent mass estimates of the massive central source by Ghez et al. (2003) and the dynamical mass estimates for the central 10 pc radius by Genzel et al. (2003),

$$
M(R)=\left(4.1+1.4 R^{1.25}\right) \times 10^{6} M_{\odot}
$$

where $R$ is radius in pc. The resulting Keplerian-like rotation of the gas disk nicely reproduces the observed features in the channel maps such as the azimuthal displacement for radially distinct features as discussed in $\S$ 4.4. To examine whether our interpretations of the gravitational potential, the geometry of the CND, and the relationship between the ionized streamers and the dense neutral gas in the CND are correct, we have created simulated channel maps of the CND using a Monte Carlo realization. They are shown side-by-side in comparison with the observed channel maps in Figure 11.

Using Matlab software package, we created a disk of 3000 particles with a $r^{-1 / 2}$ radial distribution. The model disk has a radial extent from $0.1 \mathrm{pc}$ to $2.5 \mathrm{pc}$ and has a radially dependent thickness with an opening angle of $4^{\circ}$. This particular disk geometry is adopted to allow a finite thickness which may play a role in the apparent gas distribution and kinematics in the projected view, but it is not motivated any particular observational constraints. ${ }^{3}$ The model disk is viewed with an inclination angle of $68^{\circ}$ and is rotated by $60^{\circ}$ in the sky plane to match the aspect ratio and the position angle of the CND. Each disk particle is in rotation about the central potential described by Eq. 5 and has a Gaussian random velocity component of $10 \mathrm{~km} \mathrm{~s}^{-1}$. To produce the model channel maps shown in Figure 11, model disk particles with the line-of-sight velocity matching the particular channel velocity window are selected, and their distribution is convolved with the observing beam $\left(7.0^{\prime \prime} \times 3.5^{\prime \prime}, \mathrm{PA}=-5^{\circ}\right)$. The only adjustment made to the model was adding $+13 \mathrm{~km} \mathrm{~s}^{-1}$ to the systemic velocity in order to match the highest velocity $\mathrm{HCO}^{+}$features at $-117 \mathrm{~km} \mathrm{~s}^{-1}$ and $+143 \mathrm{~km} \mathrm{~s}^{-1}$.

Even though our model disk is constructed for an illustrative purpose only and is not tuned to match the observations in detail, the side-by-side comparisons of the model and data shown in Figure 11 offer significant insights on the gas distribution and kinematics:

(i) As noted already, some of the ionized gas traced in $\mathrm{H} 41 \alpha$ is sharing the same orbital plane as the dense neutral gas in the CND (see $\S 4.4$ ). The model disk has no knowledge of temperature or ionization information, but presumably the inner part $\left(R \lesssim 30^{\prime \prime}\right)$ of the disk is either atomic or ionized. The clumps of the H41 $\alpha$ emission shown in contours on both panels in Figure 11 are frequently overlaying the model emission regions shown in grey-scale, especially in the channels with

\footnotetext{
${ }^{3}$ The only estimate found in the literature is by Latvakoski et al. (1999) of $\sim 7^{\circ}$ opening angle, based on their own morphological and dust heating model.
} 
velocities between $\pm 91 \mathrm{~km} \mathrm{~s}^{-1}$. The northern arm is sometimes modeled as being displaced from the plane defined by the CND (e.g., Latvakoski et al. 1999), but a large fraction of the northern arm gas seen in the velocity range between 0 and $+143 \mathrm{~km} \mathrm{~s}^{-1}$ closely follow our kinematical model.

(ii) The same comparison also shows that the gas in the eastern arm and the central bar do not follow the model. This leads us to believe that the ionized gas in the bar and the eastern arm is largely concentrated in a different orbital plane as suggested by others previously (e.g., Vollmer \& Duschl 2000).

(iii) The similarity between the observed $\mathrm{HCO}^{+}$emission and the model disk emission is more striking. Except for the channels with $-39 \mathrm{~km} \mathrm{~s}^{-1} \leq \mathrm{V} \leq+39 \mathrm{~km} \mathrm{~s}^{-1}$ where foreground absorption has severely altered the appearance of the emission features, most of the $\mathrm{HCO}^{+}$emission seen in the channel maps have matching features in the model. The $\mathrm{HCO}^{+}$emission arising from the CND is frequently asymmetric about its major axis, unlike the model, and the CND is probably not a solid ring or a solid disk and is likely highly clumped. Two channels show substantial $\mathrm{HCO}^{+}$ emission not predicted by our model, and these channels correspond to velocities for the " +50 $\mathrm{km} \mathrm{s}^{-1}$ streamer" and the " $+70 \mathrm{~km} \mathrm{~s}^{-1}$ feature" that are already known to deviate from the CND rotation (see below).

The $+50 \mathrm{~km} \mathrm{~s}^{-1}$ streamer is a band of emission running nearly east-west at a slightly oblique angle in the $\mathrm{V}=+39 \mathrm{~km} \mathrm{~s}^{-1}$ and $\mathrm{V}=+65 \mathrm{~km} \mathrm{~s}^{-1}$ channels, previously noted by Jackson et al. (1993) and others. This feature extends well beyond the field of view of the OVRO interferometer and is several arcmin in length in the single dish HCN (3-2) channel maps of Marshall et al. (1995). This is clearly a structure much larger than the CND in size, and thus the $+50 \mathrm{~km} \mathrm{~s}^{-1}$ streamer is most likely a foreground or a background structure, not directly associated with the CND.

The $+70 \mathrm{~km} \mathrm{~s}^{-1}$ streamer is the " 7 " shaped feature present in the $\mathrm{V}=+91 \mathrm{~km} \mathrm{~s}^{-1}$ channel. This structure is also seen in the single dish HCN (3-2) channel maps of Marshall et al. (1995), but it is more compact than the $+50 \mathrm{~km} \mathrm{~s}^{-1}$ streamer. Although this may be a chance projection, this feature lies nearly exactly over the CND. However, its velocity differs from the CND rotation by about $130 \mathrm{~km} \mathrm{~s}^{-1}$. It is not as certain that the $+70 \mathrm{~km} \mathrm{~s}^{-1}$ streamer is another foreground structure. Its long, linear appearance is suggestive of a large scale shock in origin. It is not seen in $\mathrm{H} 41 \alpha$ or in continuum to offer any further clues, and the lack of any ionized gas at the corresponding velocity is an important argument against the $+70 \mathrm{~km} \mathrm{~s}^{-1}$ streamer being part of the CND.

One of the key arguments against the CND being a coherent disk or a ring is the apparent gap along its eastern side in the previous $\mathrm{HCN}$ and $\mathrm{HCO}+$ maps. This gap now appears filled with several compact gas clumps such as the clump I in our new $\mathrm{HCO}+$ image shown in Figure 5. In addition, neutral gas with higher temperature and density also fills this gap as inferred by the observations of [C II] and $\mathrm{NH}_{3}$ (see Jackson et al. 1993; Herrnstein \& Ho 2002). Therefore earlier arguments based on the apparent morphology in the lower sensitivity $\mathrm{HCN}$ and $\mathrm{HCO}+$ maps are no longer valid. The mean velocity of the warm molecular gas found by Herrnstein \& Ho (their 
clump D) is slightly redshifted $\left(<V>\sim+50 \mathrm{~km} \mathrm{~s}^{-1}\right)$ than expected from our simple rotation model, but this velocity shift is in the correct sense if their average spectrum includes the emission from the entire north-south extent of their clump D.

In summary, we have successfully demonstrated the continuity in the distribution and kinematics of the ionized and the neutral gas within the central 3 pc diameter region surrounding Sgr $A^{\star}$ using our new observational data and a simple kinematical model. Our kinematical model reproduces many of the observed neutral and ionized gas features, as shown in Figure 11. The success of this simple model strengthens the notion that most of the gas found in the central parsec of Sgr $\mathrm{A}^{\star}$ partake in a simple rotation around the central source within the disk plane defined by the CND. Our kinematical model also identifies very clearly which gas components do not follow this simple rotation. In addition, our model suggests that some of the subtle observed effects such as the slight asymmetry between the near and far side of the disk are the products of the finite size, asymmetric observing beam and projected viewing of the 3 -D CND structure (e.g., see $V= \pm 39$ $\mathrm{km} \mathrm{s}^{-1}$ channel). The overall picture of the gas distribution and kinematics in the central $3 \mathrm{pc}$ surrounding $\operatorname{Sgr} \mathrm{A}^{\star}$ is not yet complete because information on the distribution and kinematics of atomic gas inside the CND is quite incomplete. Future arcsecond resolution imaging of dense atomic gas tracers such as [C II] will provide important clues for completing our understanding of the gaseous environment in this region.

\section{SUMMARY}

The OVRO millimeter array observations of the $\mathrm{H} 41 \alpha$ and $92 \mathrm{GHz}$ continuum emission from the ionized streamers around Sgr A* (Sgr A West) and the $\mathrm{HCO}^{+}(1-0)$ emission from the circumnuclear disk (CND) are presented along with a new survey of the hydrogen recombination lines at millimeter wavelengths ( $\mathrm{H} 31 \alpha, \mathrm{H} 41 \alpha$, and $\mathrm{H} 44 \alpha$ ) obtained using the NRAO 12-m telescope. These observations are compared with the VLA $\mathrm{H} 92 \alpha$ and the $8.3 \mathrm{GHz}$ continuum data by Roberts \& Goss (1993). We summarize our results as following:

1. The $92 \mathrm{GHz}$ continuum image is essentially identical to the VLA continuum image at $8.3 \mathrm{GHz}$ in morphology. The total continuum flux detected inside the $85^{\prime \prime}$ diameter is about $12 \mathrm{Jy}$, but some extended flux associated with structure larger than about $30^{\prime \prime}$ is missed. The brightest feature, Sgr $\mathrm{A}^{\star}$, is unresolved at $3^{\prime \prime}$ resolution of our observations and has a peak flux density of $1.95 \pm 0.30 \mathrm{Jy}$ beam $^{-1}$. Assuming the $7000 \mathrm{~K}$ electron gas fills our $0.005 \mathrm{pc}(1000 \mathrm{AU})$ beam, we infer average continuum optical depth of $\tau \lesssim 10^{-3}$ and electron density $n_{e} \sim 2 \times 10^{4} \mathrm{~cm}^{-3}$.

2. The kinematics and the morphology of H41 $\alpha$ emission are very similar to those observed in H92 $\alpha$ and $12.8 \mu \mathrm{m}$ [Ne II] lines. The brightest emission occurs along the north arm with a peak line brightness temperature of less than $10 \mathrm{~K}$. This suggests a H41 optical depth of $\tau_{L} \lesssim 10^{-3}$ averaged over the $13 \mathrm{~km} \mathrm{~s}^{-1}$ channel width. The emission along the central bar displays a line-

of-sight velocity ranging over $300 \mathrm{~km} \mathrm{~s}^{-1}$, and this gas may be located in the deepest part of the 
gravitational potential within the CND region.

3. The molecular $\mathrm{CND}$ is imaged in $\mathrm{HCO}^{+}$with a $40 \%$ improvement in resolution and $4-15$ times better sensitivity over the previous BIMA observations made in $\mathrm{HCN}$ and $\mathrm{HCO}^{+}$. The $\mathrm{CND}$ consists of several discrete concentrations, and their peak brightness ranging between 10 and 30 $\mathrm{K}$ averaged over the $0.1 \mathrm{pc}$ synthesized beam suggests that they originate from the warm (60-100 $\mathrm{K}$ ) gas responsible for $\mathrm{NH}_{3}$ and dust continuum emission in the CND. A virial analysis suggests that these clouds have mean density of $n \sim 10^{7} \mathrm{~cm}^{-3}$, sufficient to withstand the tidal shear in the region, and the CND may be a long lived structure. We estimate a total gas mass of about $3 \times 10^{5} M_{\odot}$ for the CND. Our non-detection of $\mathrm{HCO}^{+}$absorption along the Sgr A West places an upper limit to the gas column density of $\lesssim 4 \times 10^{21} \mathrm{~cm}^{-2}$, or the neutral gas within the CND is depleted of molecules.

4. A line-to-continuum ratio analysis under the LTE assumption yields a fairly uniform electron temperature of about $7000 \mathrm{~K}$ along the ionized streamers in the Sgr A West complex, in an excellent agreement with the electron temperature calculated at $8.3 \mathrm{GHz}$ using the $\mathrm{H} 92 \alpha$ observations (Roberts \& Goss 1993). Evidence for increased $T_{e}$ (or an unusually low L/C ratio) along the central bar just southwest of $\mathrm{Sgr} \mathrm{A}^{\star}$ is found in our data as well even though our data include all line emission within $\pm 350 \mathrm{~km} \mathrm{~s}^{-1}$. The low $\mathrm{L} / \mathrm{C}$ ratio we find at $92 \mathrm{GHz}$ challenges the optically thick continuum sources IRS 2 and IRS 13 as additional sources of continuum emission as suggested by Roberts, Yusef-Zadeh, \& Goss (1996). Therefore, the elevated $T_{e}$ (a lower L/C ratio) seen near the location of IRS 2 and IRS 13 is still not fully understood.

5. A comparison of the velocity integrated line flux densities for $\mathrm{H} 31 \alpha, \mathrm{H} 41 \alpha, \mathrm{H} 44 \alpha$, and $\mathrm{H} 92 \alpha$ emission from the Sgr A West complex shows line ratios characteristic of spontaneous emission from optically thin gas near LTE. A detailed analysis reveals that observed recombination line brightness is reduced by $10-30 \%$ (i.e., $b_{n} \sim 0.7-0.9$ ) from the LTE value.

6. A combined analysis of the new high resolution $\mathrm{H} 41 \alpha$ and $\mathrm{HCO}^{+}$images and a kinematic model clearly demonstrates a widely spread, close physical and dynamical link between the molecular gas in the CND and the ionized gas, including along the eastern rim where a gap in the CND was previously reported. The same analysis also clearly identifies several features that do not follow the disk rotation. We conclude that the "+50 $\mathrm{km} \mathrm{s}^{-1}$ streamer" is not directly associated with the CND. The morphology of "+70 $\mathrm{km} \mathrm{s}^{-1}$ streamer" is suggestive of its association with the CND, but its kinematics and absence of any ionized gas challenge the idea of their close association.

We are grateful to D. Roberts for providing us with the H92 $\alpha$ and $8.4 \mathrm{GHz}$ continuum data from the VLA for comparison. We are indebted to C. Lang for her expert advice and help with the GIPSY data reduction package and for many helpful suggestions as the referee of this article. In addition, we would like to acknowledge the direction gained from insightful discussions with W. M. Goss and late K. R. Anantharamaiah. We would like to thank the OVRO and the NRAO 12-m Telescope staff for the support and help without which this project would not have been possible. The 
National Radio Astronomy Observatory is a facility of the National Science Foundation operated under cooperative agreement by Associated Universities, Inc.

\section{REFERENCES}

Anantharamaiah, K. R., Zhao, J., Goss, W. M., \& Viallefond, F. 1993, ApJ, 419, 585

Anantharamaiah, K. R., Viallefond, F., Mohan, N. R., Goss, W. M., \& Zhao, J. 2000, ApJ, 537, 613

Brocklehurst, M. 1970, MNRAS, 148, 417

Brown, R. L., Lockman, F. J., \& Knapp, G. R. 1978, ARA\&A, 16, 445

Christopher, M. H., Scoville, N. Z., Stolovy, S. R., Yun, M. S. 2004, AJ, submitted.

Cocker, R. F., \& Melia, F. 1997, ApJ, 488, L149

Coil, A. L., \& Ho, P. T. P. 2000, ApJ, 533, 245

Genzel, R., Watson, D. M., Crawford, M. K., \& Townes 1985, ApJ, 297, 766

Genzel, R., \& Eckart, A. 1999, in The Galactic Center Black Hole, The ASP Conference Series, Vol. 186,3

Genzel, R., Pichon, C., Eckart, A., Gerhard, O. E., \& Ott, T. 2000, MNRAS, 317, 348

Genzel, R., Schöedel, R., Ott, T., Eisenhauer, F., Hofmann, R. et al. 2003, ApJ, 594, 812

Ghez, A. M., Morris, M., Becklin, E. E., Tanner, A., \& Kremenek, T. 2000, Nature, 407, 349

Ghez, A. M. et al. 2003, ApJ, 586, 127

Gordon, M. A. 1994, ApJ, 421, 314

Gordon, M. A., \& Walmsley, C. M. 1990, ApJ, 365, 606

Güsten, R., Genzel, R., Wright, M. C. H. Wright, Jaffe, D., Stutzki, J., \& Harris, A. I. 1987, ApJ, 318,124

Herrnstein, R. M., \& Ho, P. T. P. 2002, ApJ, 579, L83

Hogerheijde, M. R., de Geus, E. J., Spaans, M., van Langervelde, H. J., van Dishoeck, E. F. 1995, ApJ, 441, L93

Jackson, J. M., Geis, N., Genzel, R., Harris, A. I., Madden, S. et al. 1993, ApJ, 402, 173 
Kardashev, N. S. 1959, Soviet Astr., 3, 813

Lacy, J. H., Towbes, C. H., Geballe, T. R., \& Hollenbach, D. J. 1980, ApJ, 241, 132

Latvakoski, H. M., Stacy, G. J., Gull, G. E., \& Hatward, T. L. 1999, ApJ, 511, 761

Liszt, H. S., Burton, W. B., van der Hulst, J. M. 1985, A\&A, 142, 237

Lutz, D., Feuchtgruber, H., Genzel, R, Kunze, D., Rigopoulou, D. et al. 1996, A\&A, 315, L269

Maeda, Y., Baganoff, F. K., Feigelson, E. D., Morris, M., Bautz, M. W., Brandt, W. N., Burrows, D. N., Doty, J. P., Garmire, G. P., Pravdo, S. H. et al. 2002, ApJ, 570, 671

Mangum, J. G. 2000, in User's Manual for the NRAO 12 Meter Millimeter-Wave Telescope

Marr, J. M., Rudolph, A. L., Pauls, T. A., Wright, M. C. H., \& Backer, D. C. 1992, ApJL, 400, L29

Marr, J. M., Wright, M. C. H., \& Becker, D. C. 1993, ApJ, 411, 667

Marshall, J., Lesenby, A. N., \& Harris, A. I. 1995, MNRAS, 277, 594

Martin-Pintado, J., Neri, R., Planesas, P., \& Bachiller, R. 1994, A\&A, 286, 890

McGary, R. S, Coil, A. L., Ho, P. T. P. 2001, ApJ, 559, 326

Mohan, N. R., Anantharamaiah, K. R., \& W. M. Goss 2001, ApJ, 557, 659

Morris, M., \& Serabyn, E. 1996, ARAA, 34, 645

Pearson, T. J., Shepherd, M. C., Taylor, G. B., \& Myers, S. T. 1994, BAAS, 185.0808

Puxley, P. J., Mountain, C. M., Brand, P. W. J. L., Moore, T. J. T., \& Nakai, N. 1997, ApJ, 485, 143

Reid, M. J. 1989, in The Distance to the Galactic Center, IAU Symp. 136 (Kluwer Academic Publishers, Dordrecht) p.37

Roberts, D. A., Goss, W. M., van Gorkom, J. H., \& Leahy, J. P. 1991, ApJ, 366, L15

Roberts, D. A., \& Goss, W. M. 1993, ApJS, 86, 133

Roberts, D. A., Yusef-Zadeh, F., \& Goss, W. M. 1996, ApJ, 459, 627

Sanders, R. H. 1998, MNRAS, 294, 35

Schöedel, R., Ott, T., Genzel, R., Eckart, A., Mouawad, N., Alexander, T. 2003, ApJ, 596, 1015

Schwarz, U. J., Bregman, J. D., \& van Gorkom, J. H. 1989, A\&A, 215, 33 
Scoville, N. Z., Carlstrom, J. E., Chandler, C. J., Phillips, J. A., Scott, S. L., Tilanus, R. P. J., \& Wang, Z. 1993, PASP, 105, 1482

Scoville, N. Z., Stolovy, S. R., Rieke, M., Christopher, M., \& Yusef-Zadeh, F. 2003, ApJ, 594, 294

Seaquist, E. R., Kerton, C. R., \& Bell, M. B. 1994, ApJ, 429, 612

Seaquist, E. R., Carlstrom, J. E., Byrant, \& Bell, M. B. 1996, ApJ, 465, 691

Serabyn, E., \& Lacy, J. H. 1985, ApJ, 293, 445

Serabyn, E., Lacy, J. H., Townes, C. H., \& Bharat, R. 1988, ApJ, 326, 171

Serabyn, E., Carlstrom, J., Lay, O., Lis, D. C., Hunter, T. R., Lacy, J. H., \& Hills, R. E. 1997, ApJ, 490, 177

Sternberg, A., Dalgarno, A. 1989, ApJ, 338, 197

Telesco, C. M., Davidson, J. A., \& Werner, M. W. 1996, ApJ, 456, 541

Tsuboi, M., Handa, T., \& Ukita, N. 1999, ApJS, 120, 1

Vollmer, B., \& Duschl, W. 2000, New Astronomy, 4, 581

Vollmer, B., \& Duschl, W. 2002, A\&A, 388, 128

Walmsley, C. M. 1990, A\&AS, 82, 201

Wright, M. C. H., \& Backer, D. C. 1993, ApJ, 417, 560

Wright, M. C. H., Coil, A. L., McGary, R. S., Ho, P. T. P., \& Harris, A. I. 2001, ApJ, 551, 254

Yun, M. S., Scoville, N. Z., Shukla, H. 2003, in The Neutral ISM in Starburst Galaxies, ASP Conference Series, in press

Yusef-Zadeh, F., Morris, M., Ekers, R. D. 1990, Nature, 348, 45

Yusef-Zadeh, F., Melia, F., \& Wardle, M. 2000, Science, 287, 85

Zhao, J., Anantharamaiah, K. R. Goss, W. M., \& Villefond, F. 1996, ApJ, 472, 54

Zhao, J., \& Goss, W. M. 1999, ASP Conf. Series, 186, 224 


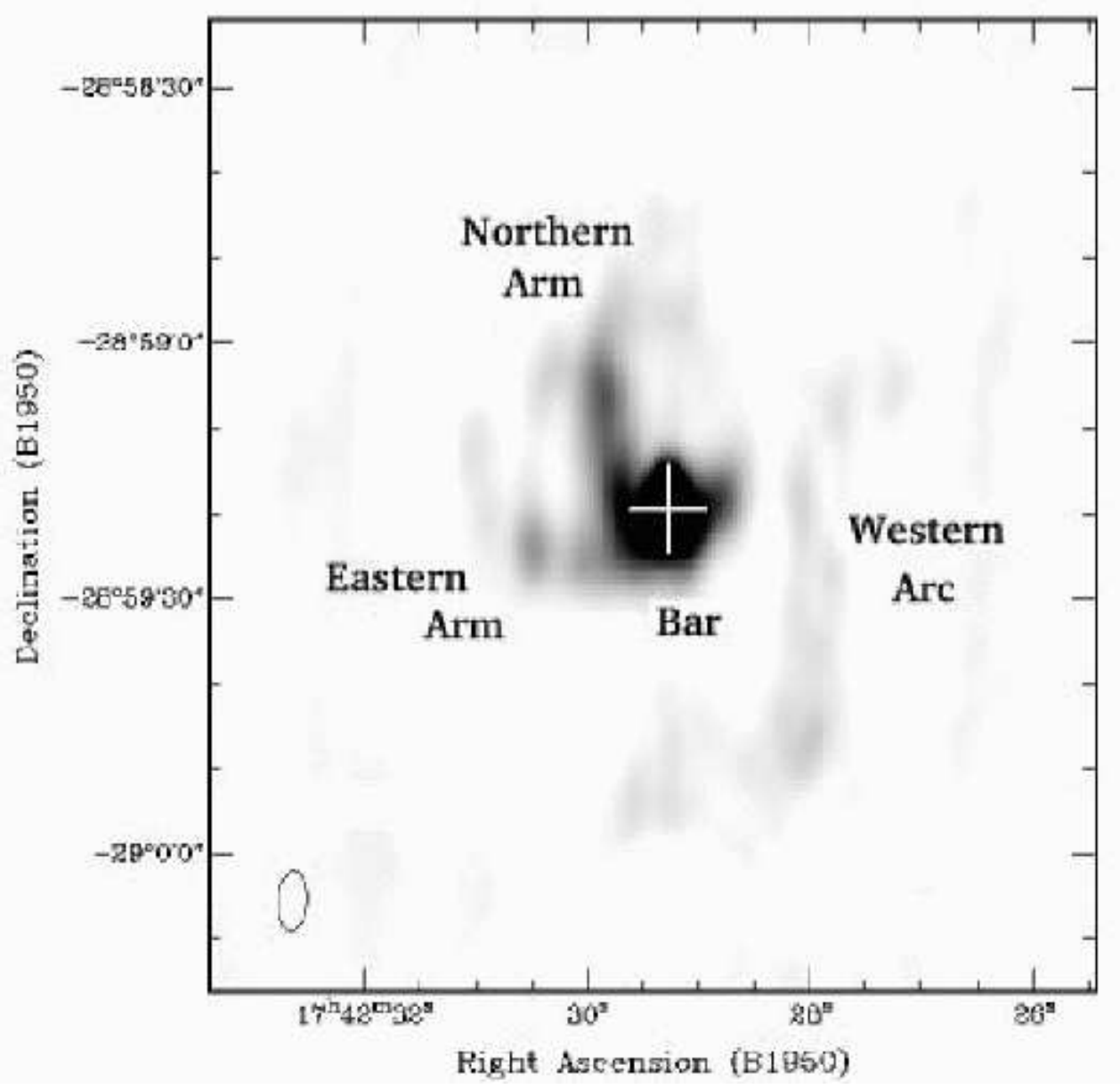

Fig. 1. - Deconvolved image of $92 \mathrm{GHz}$ continuum emission from Sgr A complex at $6.95^{\prime \prime} \times 3.47^{\prime \prime}$ $\left(\mathrm{PA}=-5^{\circ}\right)$ resolution of the synthesized beam. The cross marks the position of Sgr $\mathrm{A}^{\star}$. The grey-scale range is between 0.03 to $0.40 \mathrm{Jy}_{\text {beam }}{ }^{-1}$ with RMS noise of $0.01 \mathrm{Jy}^{\mathrm{beam}}{ }^{-1}$. 


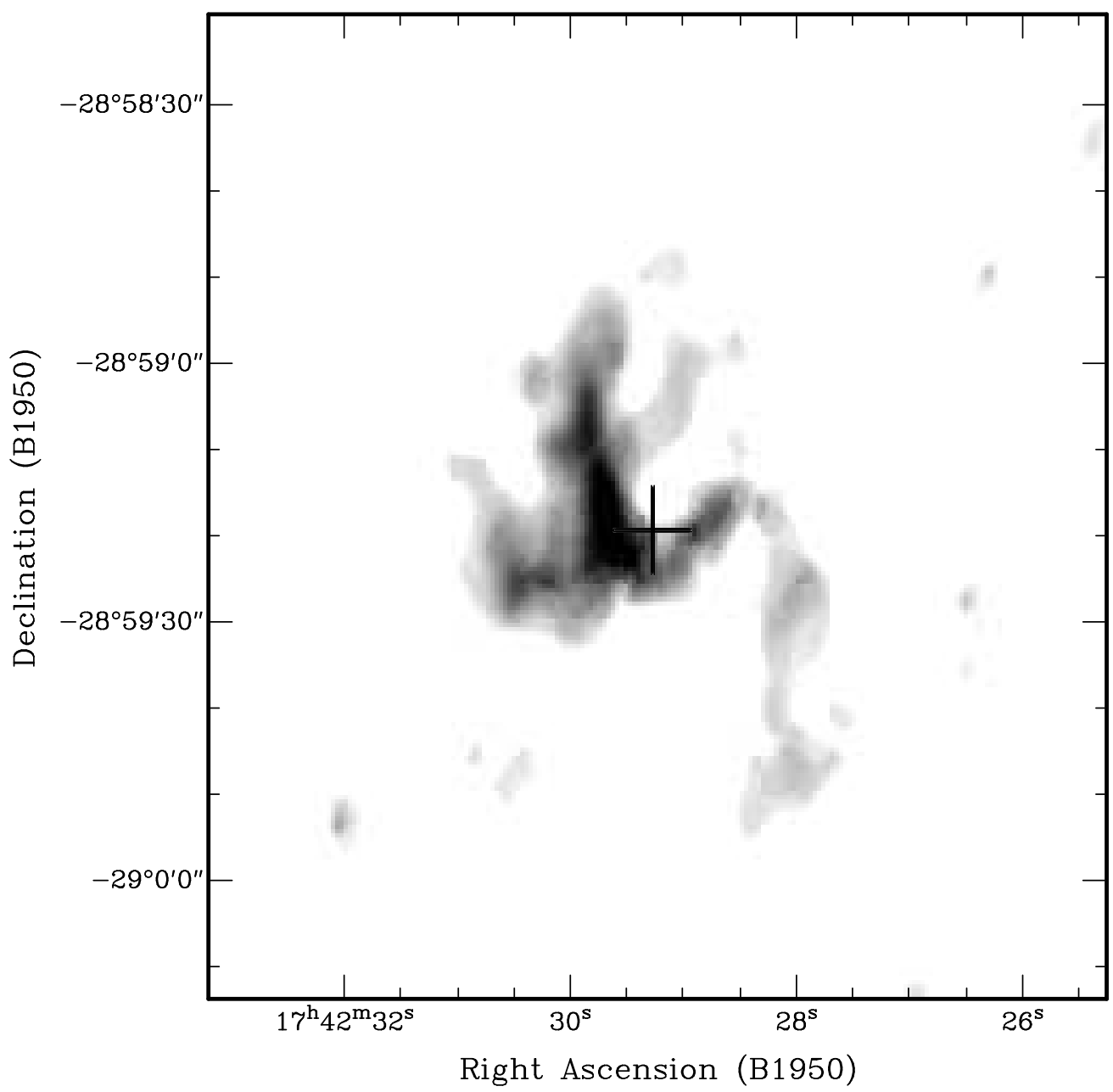

Fig. 2.- Integrated line emission of the H $41 \alpha$ line from Sgr A complex at $6.95^{\prime \prime} \times 3.47^{\prime \prime}(\mathrm{PA}=$ $\left.-5^{\circ}\right)$ resolution of the synthesized beam. The cross marks the position of Sgr $\mathrm{A}^{\star}$. The grey-scale range is between 0 and $12 \mathrm{Jy} \mathrm{km} \mathrm{s}^{-1}$ beam $^{-1}$.

Fig. 3.- (a) Channel maps (from $-221 \mathrm{~km} \mathrm{~s}^{-1}$ to $-13 \mathrm{~km} \mathrm{~s}^{-1}$ ) of $\mathrm{HCO}^{+}$emission are shown in grey-scale and H41 $\alpha$ in contours. (b) Channel maps (from $+13 \mathrm{~km} \mathrm{~s}^{-1}$ to $+221 \mathrm{~km} \mathrm{~s}^{-1}$ ) of $\mathrm{HCO}^{+}$emission are shown in grey-scale and $\mathrm{H} 41 \alpha$ in contours. The grey-scale ranges between -100 (white) and +500 (black) mJy beam ${ }^{-1}$ for $\mathrm{HCO}^{+}$. The contour levels are $-2,+2,+3,+4,+5$, and +6 times 15 mJy beam ${ }^{-1}(1 \sigma)$. The location of $\mathrm{Sgr} \mathrm{A}^{\star}$ is marked at the center with a cross. 

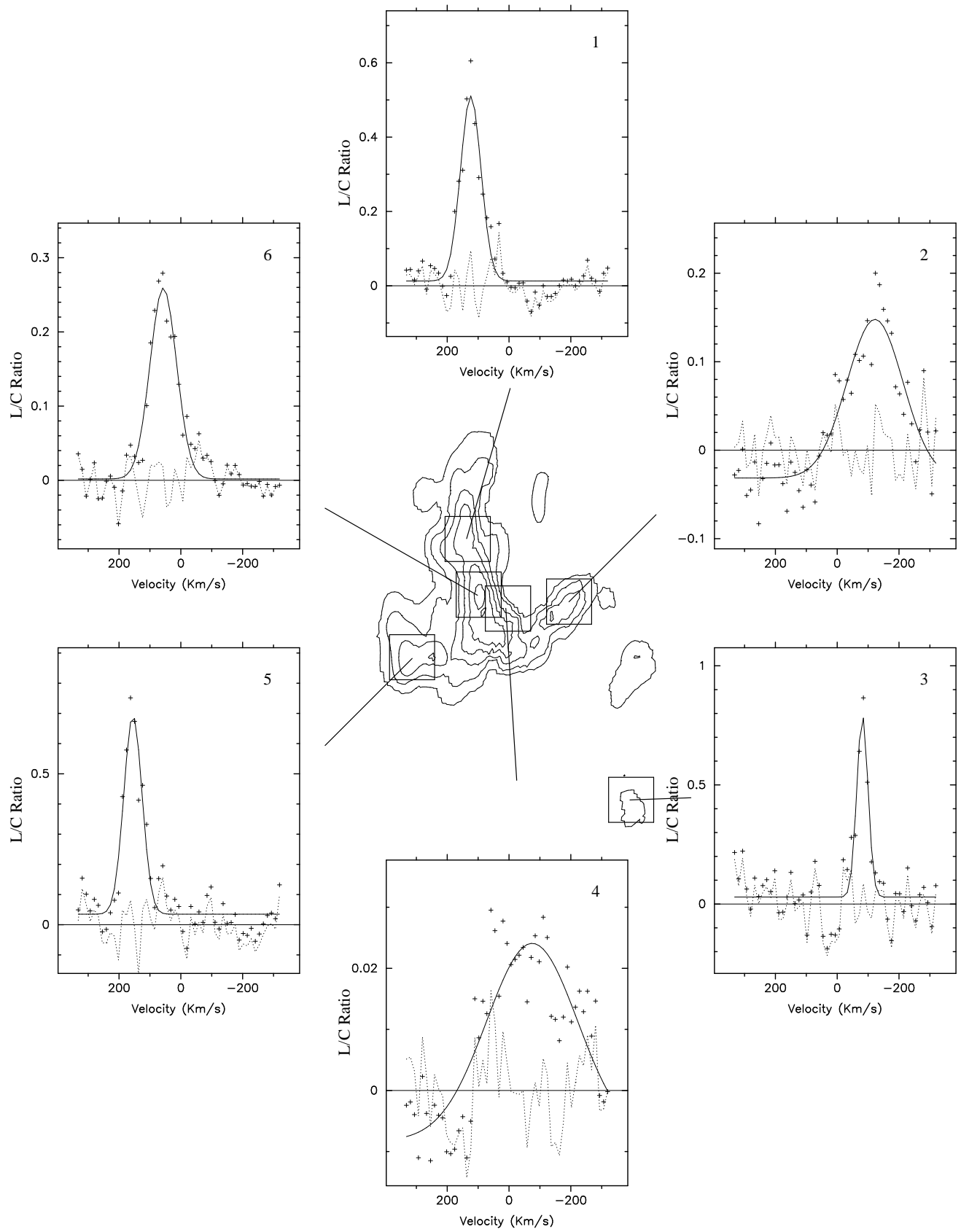

Fig. 4.- A contour map of $\mathrm{H} 41 \alpha$ emission is shown with line-to-continuum (L/C) plots of respective regions. The contour levels are $+1,+2,+3,+4,+5$, and +6 times $2.3 \mathrm{Jy} \mathrm{km} \mathrm{s}^{-1}$. Ratios of the $\mathrm{L} / \mathrm{C}$ are marked on the $\mathrm{y}$-axis and the velocity ranges in $\mathrm{km} \mathrm{s}^{-1}$ are shown on the $\mathrm{x}$-axis. Crosses mark the data points, solid line represents the model Gaussian fit, and the dashed line represents the residual. 


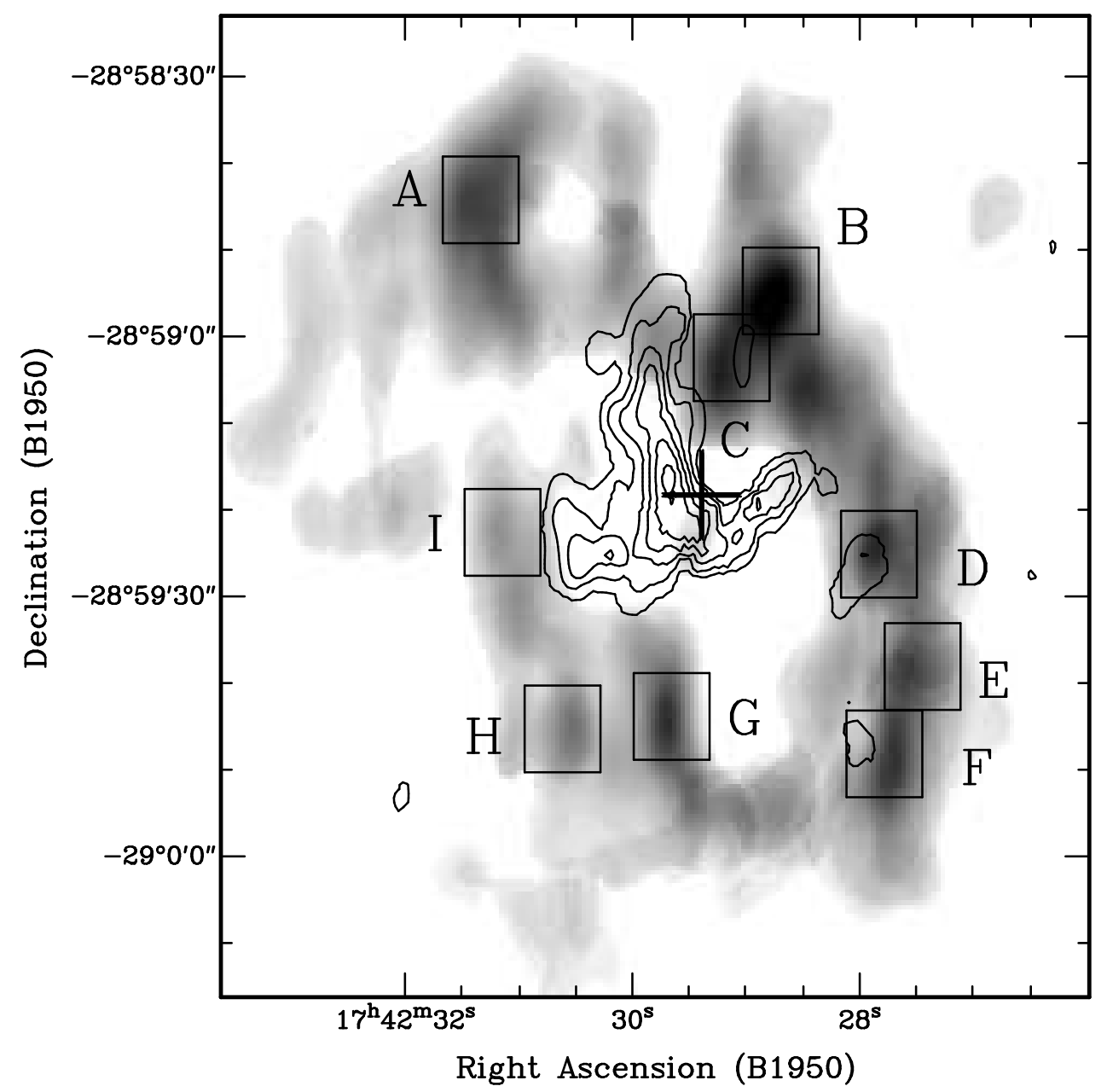

Fig. 5.- Integrated line emission of the $\mathrm{HCO}^{+}$line from Sgr A complex at $6.95^{\prime \prime} \times 3.47^{\prime \prime}(\mathrm{PA}=$ $\left.-5^{\circ}\right)$ resolution. The cross marks the position of $\mathrm{Sgr} \mathrm{A}^{\star}$. The grey-scale range is between 7 to 32 Jy km s$~^{-1}$ beam $^{-1}$. Superimposed are the contours of $\mathrm{H} 41 \alpha$ emission. The contour levels are 2.3, 4.6, 6.9, 9.2, 11.6, and $13.9 \mathrm{Jy} \mathrm{km} \mathrm{s}^{-1}$ beam $^{-1}$. The boxes mark the dense gas clumps described in Table 5 . 


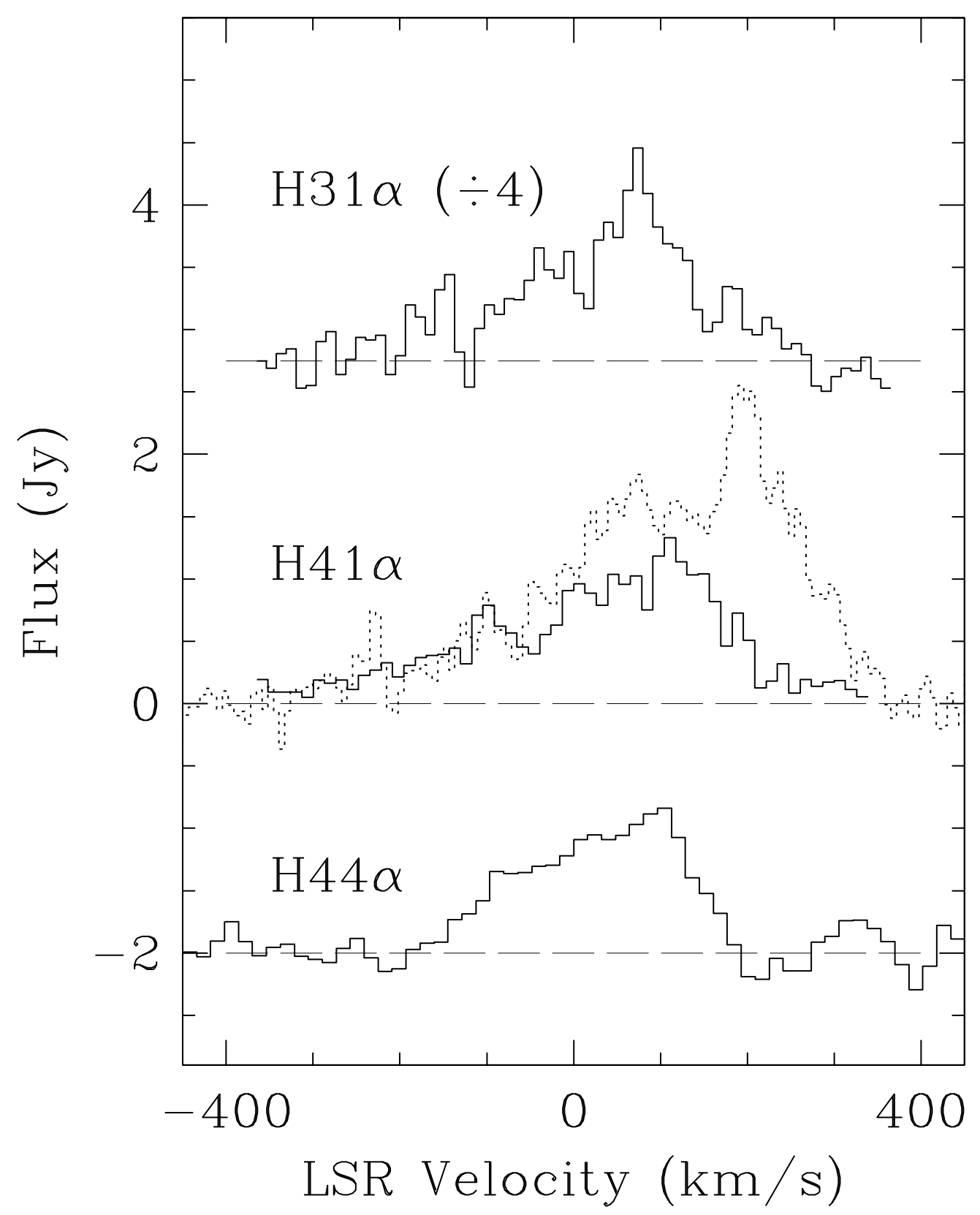

Fig. 6.- H31 $\alpha, \mathrm{H} 41 \alpha$, and $\mathrm{H} 44 \alpha$ spectra of Sgr A West obtained with the NRAO 12-m telescope. The H31 $\alpha$ spectrum is made by summing 8 independent spectra covering the whole Sgr A West, scaled down by a factor of four. Both the OVRO (solid line) and the NRAO 12-m (dotted line) spectrum are shown for $\mathrm{H} 41 \alpha$ line. The excess emission in the NRAO 12-m spectrum near +200 $\mathrm{km} \mathrm{s}^{-1}$ may be due the contamination by widely distributed foreground $\mathrm{CH}_{3} \mathrm{CN}$ emission (see text). 


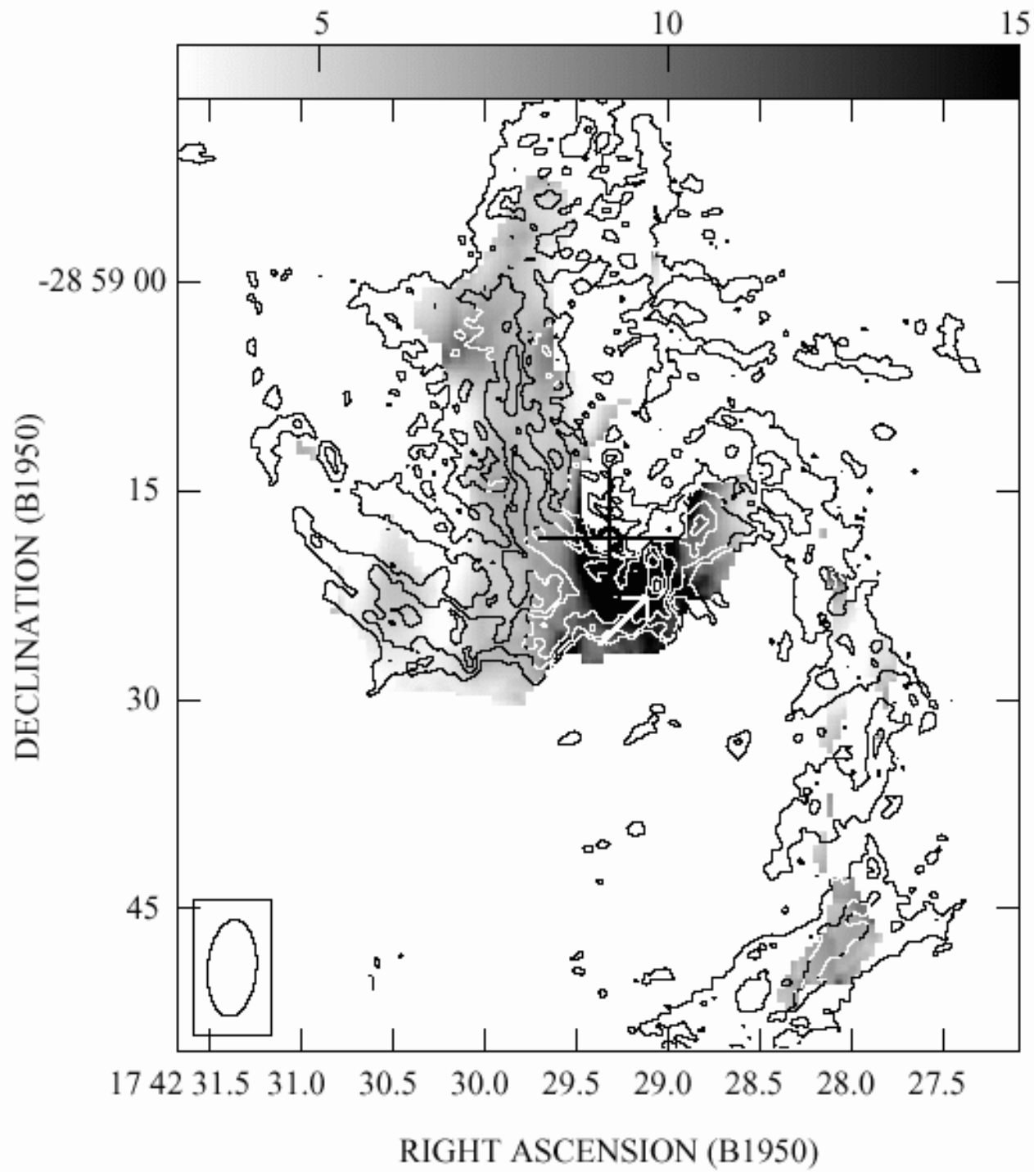

Fig. 7.- Grey-scale representation of LTE electron temperature distribution in Sgr A West. The grey-scale ranges between 3000 to $15000 \mathrm{~K}$. The $8.3 \mathrm{GHz}$ continuum image at $0.62^{\prime \prime} \times 0.51^{\prime \prime}$ resolution by Roberts \& Goss (1993) is shown in contours. The contour levels are 2.5, 5, 10, 20, 30, 40, 50, and $60 \mathrm{mJy}^{\text {beam }}{ }^{-1}$. The thick cross marks the position of Sgr $\mathrm{A}^{\star}$. The location of the IR source IRS 2 is shown with a thick white arrow, and the second continuum source about $2^{\prime \prime}$ to the north is IRS 13 . 


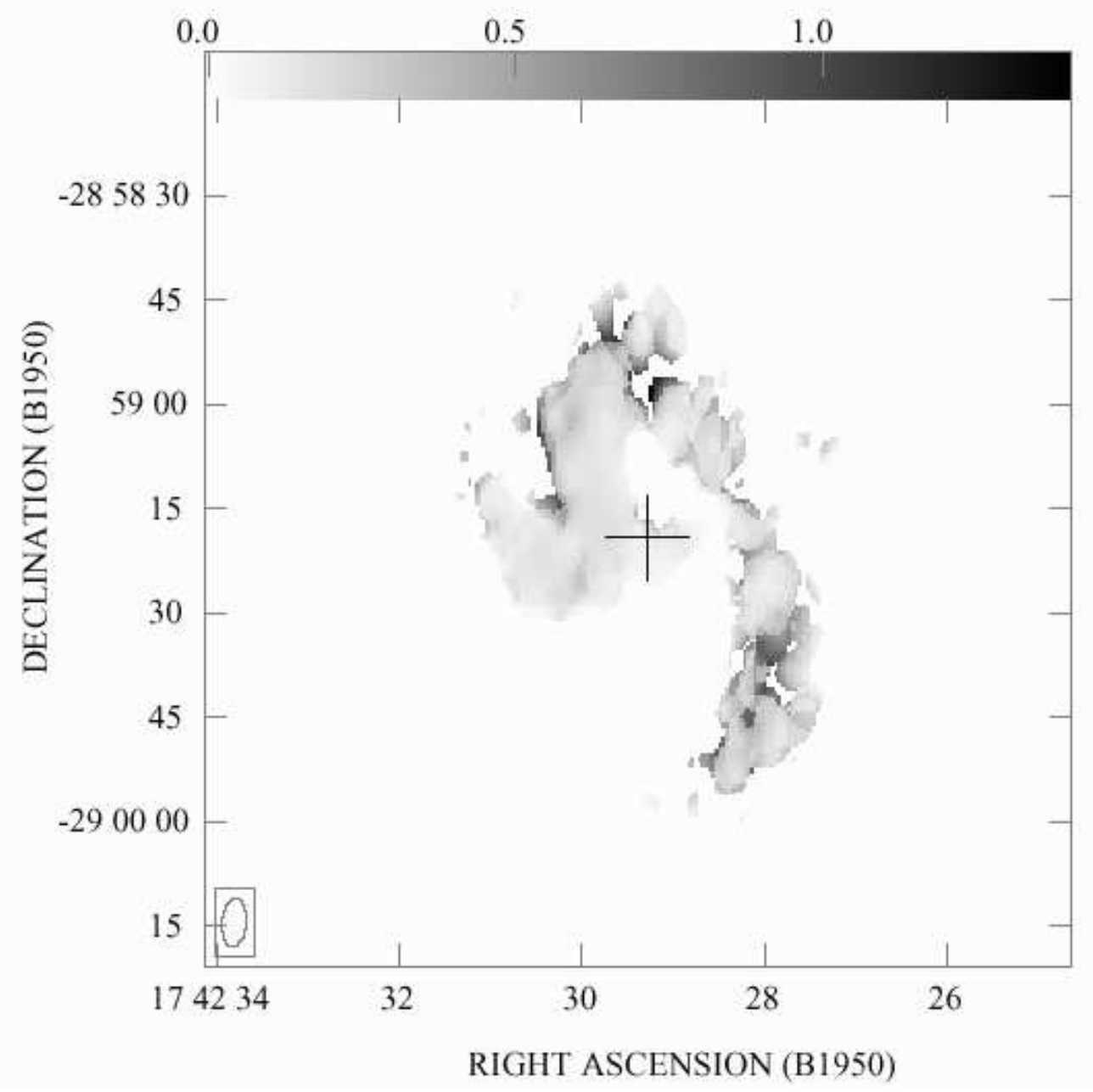

Fig. 8. - A ratio map between $\mathrm{H} 92 \alpha$ and $\mathrm{H} 41 \alpha$ line emission is shown in grey-scale (from 0.0 to 1.5). The integrated H92 $\alpha$ line data from Roberts et al. (1993) were convolved with the OVRO beam. The ratio is nearly constant, $\sim 0.15$, in most regions. 


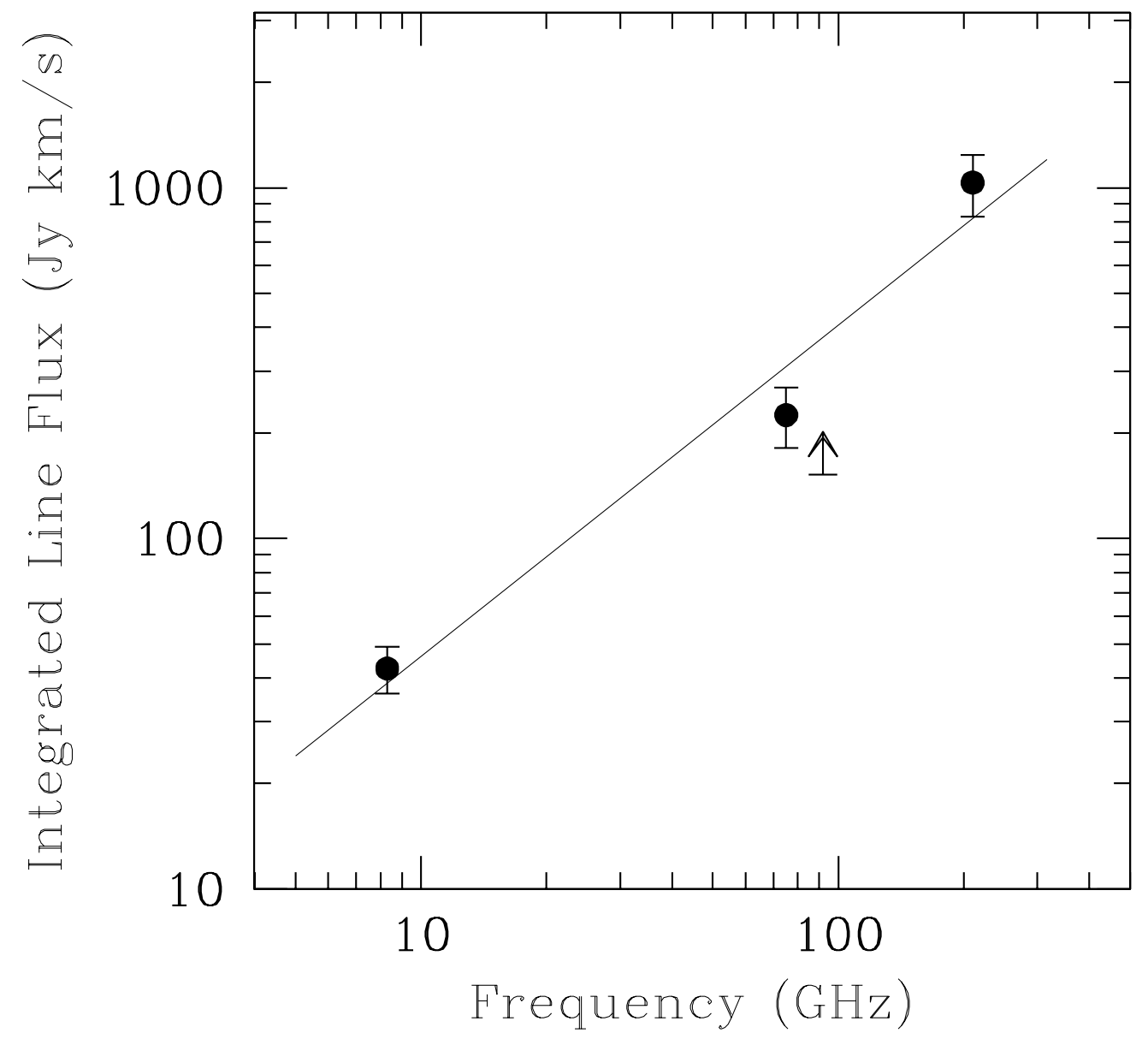

Fig. 9.- Velocity integrated line flux densities of $\mathrm{H} 31 \alpha, \mathrm{H} 44 \alpha$, and $\mathrm{H} 92 \alpha$ are plotted as a function of frequency. The H41 $\alpha$ measurement from the OVRO data is shown only as an upper limit since some of the extended emission is resolved out by the interferometer. The solid line is the best fit power-law relation, $S_{L} \propto \nu^{\alpha}$. The slope $\alpha=0.95 \pm 0.18$ is very close to the theoretical value for spontaneous emission $\left(S_{L} \propto \nu^{+1}\right)$. 

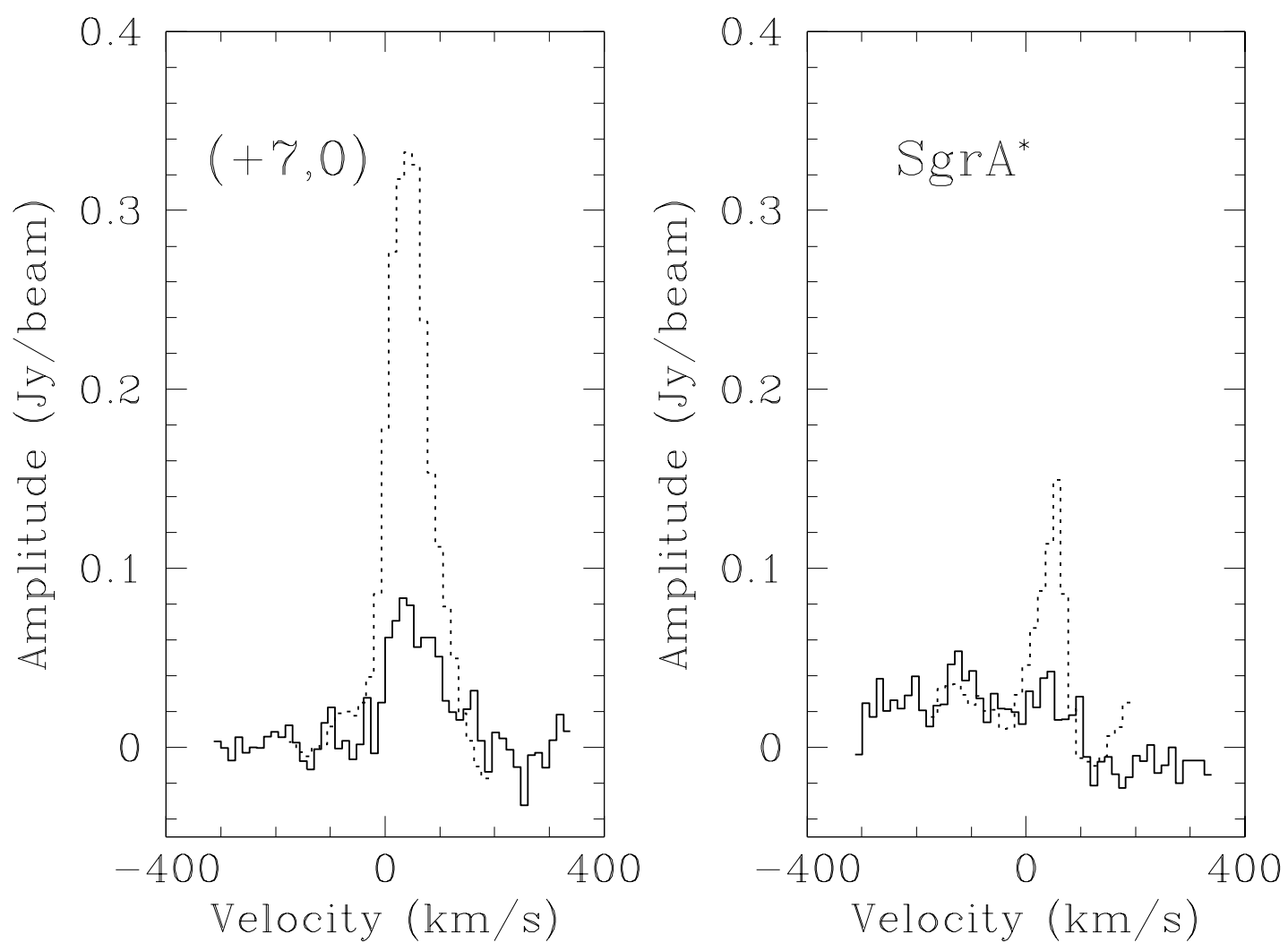

Fig. 10.- A comparison of the H41 $\alpha$ (solid line) and H92 $\alpha$ (dotted line, multiplied by a factor of 15) spectrum, one at the Sgr $\mathrm{A}^{\star}$ position (right) and at an adjacent position $7^{\prime \prime}$ to the east (left).

Fig. 11. - (a) Channel maps (from $-195 \mathrm{~km} \mathrm{~s}^{-1}$ to $-13 \mathrm{~km} \mathrm{~s}^{-1}$ ) of $\mathrm{HCO}^{+}$emission are shown in grey-scale. In each twin panel plot, the observed $\mathrm{HCO}^{+}$emission is shown on the left panel while the simulated disk model (see $\S 4.5$ ) is shown on the right panel. The contours of H41 $\alpha$ emission are shown in both panels. The grey-scale ranges between -100 (white) and +500 (black) $\mathrm{mJy} \mathrm{beam}^{-1}$ for $\mathrm{HCO}^{+}$. The greyscale for the model shown on the right panel uses the full range in model units. The contour levels are $-2,+2,+3,+4,+5$, and +6 times 15 mJy beam ${ }^{-1}(1 \sigma)$. Each box is $100^{\prime \prime}$ on each side, and Sgr $A^{\star}$ is located at the center. (b) Channel maps (from $+13 \mathrm{~km} \mathrm{~s}^{-1}$ to +195 $\mathrm{km} \mathrm{s}^{-1}$ ) of $\mathrm{HCO}^{+}$in grey-scale superimposed with contours of $\mathrm{H} 41 \alpha$. 
Table 1. Summary of the OVRO Observations

\begin{tabular}{lc}
\hline \hline & \\
\hline Rest Frequency of $\mathrm{H} 41 \alpha$ line & $92.034 \mathrm{GHz}$ \\
Rest Frequency of $\mathrm{HCO}^{+}(J=1 \rightarrow 0)$ line & $89.188 \mathrm{GHz}$ \\
Primary beam $(\mathrm{HPBW})$ & $85^{\prime \prime}$ \\
Phase center & $\alpha(B 1950)=17^{h} 51^{m} 29.4^{s}$ \\
& $\delta(B 1950)=-28^{\circ} 59^{\prime} 19.2^{\prime \prime}$ \\
Center LSR velocity & $0.0 \mathrm{~km} \mathrm{~s}^{-1}$ \\
Synthesized beam $(\mathrm{HPBW})$ & $6.95^{\prime \prime} \times 3.47^{\prime \prime}\left(\mathrm{PA}=-5^{\circ}\right)$ \\
Spectrometer settings & $60 \times 4 \mathrm{MHz}\left(13 \mathrm{~km} \mathrm{~s}^{-1}\right)$ \\
RMS noise & $0.13 \mathrm{Jy} \mathrm{beam}{ }^{-1}(0.21 \mathrm{~K})$ \\
\hline
\end{tabular}

Table 2. $92 \mathrm{GHz}$ continuum emission

\begin{tabular}{lllll}
\hline \hline \multicolumn{1}{c}{ Feature } & $\begin{array}{c}\text { Size } \\
(\mathrm{pc})\end{array}$ & $\begin{array}{c}S_{\text {peak }} \\
\left(\mathrm{Jy} \mathrm{Beam}^{-1}\right)\end{array}$ & $\begin{array}{c}T_{\text {peak }} \\
(\mathrm{K})\end{array}$ & $\begin{array}{c}S_{\text {total }} \\
(\mathrm{Jy})\end{array}$ \\
\hline Northern Arm & 0.9 & $0.39 \pm 0.07$ & $3.1 \pm 0.6$ & $1.09 \pm 0.16$ \\
Sgr A $^{\star}$ & 0.1 & $1.95 \pm 0.29$ & $15.6 \pm 2.3$ & $1.95 \pm 0.30$ \\
Bar & 1.1 & $0.75 \pm 0.14$ & $6.0 \pm 1.1$ & $0.90 \pm 0.29$ \\
Western Arc & 1.5 & $0.14 \pm 0.02$ & $1.1 \pm 0.2$ & $1.08 \pm 0.08$ \\
Eastern Arm & 0.4 & $0.29 \pm 0.05$ & $2.3 \pm 0.4$ & $0.52 \pm 0.12$ \\
\hline
\end{tabular}

Table 3. A Summary of H41 $\alpha$ line intensity

\begin{tabular}{lcccc}
\hline \hline \multicolumn{1}{c}{ Region } & $\begin{array}{c}\text { Size } \\
(\mathrm{pc})\end{array}$ & $\begin{array}{c}S_{\text {peak }} \\
\left(\mathrm{Jy} \mathrm{Beam}^{-1}\right)\end{array}$ & $\begin{array}{c}T_{\text {peak }} \\
(\mathrm{K})\end{array}$ & $\begin{array}{c}S_{\text {total }} \\
(\mathrm{Jy})\end{array}$ \\
\hline Northern Arm & 0.9 & $1.18 \pm 0.27$ & $9.4 \pm 2.2$ & $4.70 \pm 0.71$ \\
Bar & 1.1 & $1.12 \pm 0.21$ & $9.0 \pm 1.7$ & $5.70 \pm 0.37$ \\
Western Arc & 1.5 & $0.50 \pm 0.14$ & $4.0 \pm 1.1$ & $1.86 \pm 0.39$ \\
Eastern Arm & 0.4 & $0.90 \pm 0.15$ & $7.2 \pm 1.2$ & $2.02 \pm 0.36$ \\
\hline
\end{tabular}


Table 4. LTE electron temperature of the regions identified in Figure 4

\begin{tabular}{|c|c|c|c|c|c|}
\hline Region & $\begin{array}{c}\text { Box Center } \\
\text { (J2000) }\end{array}$ & $\begin{array}{c}V_{L S R} \\
\left(\mathrm{~km} \mathrm{~s}^{-1}\right)\end{array}$ & $\begin{array}{c}\Delta V \\
\left(\mathrm{~km} \mathrm{~s}^{-1}\right)\end{array}$ & $<T_{L} / T_{C}>$ & $\begin{array}{c}\text { Temperature } T_{e}^{\star} \\
(\mathrm{K})\end{array}$ \\
\hline 1 & $17^{h} 42^{m} 29 .^{s} 71$ & $+114 \pm 3$ & $78 \pm 5$ & $0.50 \pm 0.04$ & $6394 \pm 608$ \\
\hline 2 & $17^{h} 42^{m} 28 .^{s} 60$ & $-132 \pm 8$ & $213 \pm 18$ & $0.18 \pm 0.02$ & $6473 \pm 891$ \\
\hline 3 & $17^{h} 42^{m} 27 .{ }^{s} 92$ & $-92 \pm 3$ & $43 \pm 5$ & $0.80 \pm 0.13$ & $7470 \pm 1372$ \\
\hline 4 & $17^{h} 42^{m} 29 .{ }^{s} 27$ & $-117 \pm 20$ & $347 \pm 48$ & $0.03 \pm 0.01$ & $18806 \pm 4770$ \\
\hline 5 & $17^{h} 42^{m} 30 .^{s} 32$ & $+142 \pm 3$ & $70 \pm 5$ & $0.66 \pm 0.07$ & $5528 \pm 623$ \\
\hline 6 & $17^{h} 42^{m} 29 .^{s} 59$ & $+46 \pm 3$ & $101 \pm 6$ & $0.26 \pm 0.02$ & $9057 \pm 825$ \\
\hline
\end{tabular}

Table 5. $\mathrm{HCO}^{+}$line intensity of the nine brightest clumps in the CND

\begin{tabular}{ccccccc}
\hline \hline Clump & $\begin{array}{c}\text { Size } \\
(\mathrm{pc})\end{array}$ & $\begin{array}{c}S_{\text {peak }} \\
\left(\mathrm{Jy} \mathrm{Beam}^{-1}\right)\end{array}$ & $\begin{array}{c}T_{\text {peak }} \\
(\mathrm{K})\end{array}$ & $\begin{array}{c}\Delta V \\
\left(\mathrm{~km} \mathrm{~s}^{-1}\right)\end{array}$ & $\begin{array}{c}M_{\text {vir }} \\
\left(\mathrm{M}_{\odot}\right)\end{array}$ & $\begin{array}{c}\bar{n}_{\text {vir }} \\
\left(\mathrm{cm}^{-3}\right)\end{array}$ \\
\hline & & & & & \\
\hline $\mathrm{A}$ & 0.4 & $3.5 \pm 0.8$ & $27.9 \pm 6.4$ & 20.7 & $2.1 \times 10^{4}$ & $1.4 \times 10^{7}$ \\
$\mathrm{~B}$ & 0.3 & $1.6 \pm 0.4$ & $12.7 \pm 3.2$ & 17.3 & $1.1 \times 10^{4}$ & $1.8 \times 10^{7}$ \\
$\mathrm{C}$ & 0.3 & $1.0 \pm 0.8$ & $8.0 \pm 6.4$ & 12.0 & $0.5 \times 10^{4}$ & $0.9 \times 10^{7}$ \\
$\mathrm{D}$ & 0.4 & $1.2 \pm 0.3$ & $9.5 \pm 2.3$ & 29.9 & $4.5 \times 10^{4}$ & $3.0 \times 10^{7}$ \\
$\mathrm{E}$ & 0.2 & $1.5 \pm 0.9$ & $11.9 \pm 7.2$ & 11.6 & $0.3 \times 10^{4}$ & $1.8 \times 10^{7}$ \\
$\mathrm{~F}$ & 0.3 & $1.9 \pm 0.4$ & $15.1 \pm 3.2$ & 19.4 & $1.4 \times 10^{4}$ & $2.2 \times 10^{7}$ \\
$\mathrm{G}$ & 0.6 & $1.5 \pm 0.4$ & $12.0 \pm 3.2$ & 16.1 & $1.9 \times 10^{4}$ & $0.4 \times 10^{7}$ \\
$\mathrm{H}$ & 0.4 & $1.0 \pm 0.5$ & $8.0 \pm 4.0$ & 19.0 & $1.8 \times 10^{4}$ & $1.2 \times 10^{7}$ \\
$\mathrm{I}$ & 0.2 & $1.8 \pm 0.7$ & $14.3 \pm 5.6$ & 13.0 & $0.4 \times 10^{4}$ & $2.3 \times 10^{7}$ \\
\hline
\end{tabular}


Table 6. Summary of the NRAO 12-m Observations

\begin{tabular}{ccc}
\hline \hline Line & $\begin{array}{c}\nu \\
(\mathrm{GHz})\end{array}$ & $\begin{array}{c}S \Delta V \\
\left(\mathrm{Jy} \mathrm{km} \mathrm{s}^{-1}\right)\end{array}$ \\
\hline $\mathrm{H} 31 \alpha$ & 210.502 & $1034 \pm 207$ \\
$\mathrm{H} 35 \alpha$ & 146.969 & - \\
$\mathrm{H} 41 \alpha$ & 92.034 & - \\
$\mathrm{H} 44 \alpha$ & 74.645 & $232 \pm 46$ \\
$\mathrm{H} 92 \alpha$ & 8.309 & $42.6 \pm 6.5$ \\
\hline
\end{tabular}


This figure "f3a.jpg" is available in "jpg" format from: http://arXiv.org/ps/astro-ph/0408136v1 
This figure "f3b.jpg" is available in "jpg" format from: http://arXiv.org/ps/astro-ph/0408136v1 
This figure "f11a.jpg" is available in "jpg" format from: http://arXiv.org/ps/astro-ph/0408136v1 
This figure "f11b.jpg" is available in "jpg" format from: http://arXiv.org/ps/astro-ph/0408136v1 